\title{
A long-term numerical solution for the insolation quantities of the Earth
}

\author{
J. Laskar ${ }^{1}$, P. Robutel ${ }^{1}$, F. Joutel ${ }^{1}$, M. Gastineau ${ }^{1}$, A. C. M. Correia ${ }^{1,2}$, and B. Levrard ${ }^{1}$ \\ 1 Astronomie et Systèmes Dynamiques, IMCCE - CNRS UMR8028, 77 Av. Denfert-Rochereau, 75014 Paris, France \\ e-mail: laskar@imcce.fr \\ 2 Departamento de Física da Universidade de Aveiro, Campus Universitário de Santiago, 3810-193 Aveiro, Portugal
}

Received 23 May 2004 / Accepted 11 August 2004

\begin{abstract}
We present here a new solution for the astronomical computation of the insolation quantities on Earth spanning from -250 Myr to 250 Myr. This solution has been improved with respect to La93 (Laskar et al. 1993) by using a direct integration of the gravitational equations for the orbital motion, and by improving the dissipative contributions, in particular in the evolution of the Earth-Moon System. The orbital solution has been used for the calibration of the Neogene period (Lourens et al. 2004), and is expected to be used for age calibrations of paleoclimatic data over 40 to 50 Myr, eventually over the full Palaeogene period (65 Myr) with caution. Beyond this time span, the chaotic evolution of the orbits prevents a precise determination of the Earth's motion. However, the most regular components of the orbital solution could still be used over a much longer time span, which is why we provide here the solution over 250 Myr. Over this time interval, the most striking feature of the obliquity solution, apart from a secular global increase due to tidal dissipation, is a strong decrease of about 0.38 degree in the next few millions of years, due to the crossing of the $s_{6}+g_{5}-g_{6}$ resonance (Laskar et al. 1993). For the calibration of the Mesozoic time scale (about 65 to $250 \mathrm{Myr}$ ), we propose to use the term of largest amplitude in the eccentricity, related to $g_{2}-g_{5}$, with a fixed frequency of $3.200^{\prime \prime} / \mathrm{yr}$, corresponding to a period of $405000 \mathrm{yr}$. The uncertainty of this time scale over $100 \mathrm{Myr}$ should be about $0.1 \%$, and $0.2 \%$ over the full Mesozoic era.
\end{abstract}

Key words. chaos - celestial mechanics - ephemerides - Earth

\section{Introduction}

Due to gravitational planetary perturbations, the elliptical elements of the orbit of the Earth are slowly changing in time, as is the orientation of the planet's spin axis. These changes induce variations of the insolation received on the Earth's surface. The first computation of the secular variations of the Earth's orbital elements were made by Lagrange $(1781,1782)$, and then Pontécoulant (1834), but it was the work of Agassiz (1840), showing geological evidence of past ice ages, that triggered the search for a correlation between the geological evidence of large climatic changes, and the variations of the Earth's astronomical parameters. Shortly after, Adhémar (1842) proposed that these climatic variations originated from the precession of the Earth's rotation axis.

After the publication of a more precise solution of the Earth by Le Verrier (1856), that took into account the secular perturbations of all main planets except for Neptune, Croll (1890) proposed that the variation of the Earth's eccentricity was also an important parameter for the understanding of the past climates of the Earth.

The first computations of the variations of the obliquity (angle between the equator and orbital plane) due to the secular variations of the orbital plane of the Earth are due to
Pilgrim (1904), and were later used by Milankovitch (1941) to establish his theory of the Earth's insolation parameters. Since then, the understanding of the climate response to the orbital forcing has evolved, but all the necessary ingredients for the insolation computations were present in Milankovitch's work.

The revival of the Milankovitch theory of paleoclimate can be related to the landmark work of Hays et al. (1976), that established a correlation between astronomical forcing and the $\delta^{18} \mathrm{O}$ records over the past $500 \mathrm{kyr}$. The Milankovitch theory has since been confirmed overall with variations in the climate response to the insolation forcing (see Imbrie \& Imbrie 1979; Imbrie 1982, for more historical details; and Zachos et al. 2001; Grastein et al. 2004, for a recent review on the astronomical calibration of geological data).

Since the work of Pilgrim (1904) and Milankovitch (1941), the orbital and precession quantities of the Earth have undergone several improvements. Le Verrier's solution (1856) consisted in the linearized equations for the secular evolution of the planetary orbits. Stockwell (1873), and Harzer (1895) added the planet Neptune to Le Verrier's computations. A significant improvement is due to Hill (1897) who discovered that the proximity of a resonance in Jupiter and Saturn's motion induces some important complements at the second order with respect to the masses. The solution of 
Brouwer \& Van Woerkom (1950) is essentially the solution of Le Verrier and Stockwell, complemented by the higher order contributions computed by Hill. This solution was used for insolation computations by Sharav \& Boudnikova (1967a,b) with updated values of the parameters, and later on by Vernekar (1972). The computations of Vernekar were actually used by Hays et al. (1976).

The next improvement in the computation of the orbital data is by Bretagnon (1974), who computed the terms of second order and degree 3 in eccentricity and inclination in the secular equations, but omitted the terms of degree 5 in the JupiterSaturn system from Hill (1897). This solution was then used by Berger (1978) for the computation of the precession and insolation quantities of the Earth, following Sharav \& Boudnikova (1967a,b). All these works assumed implicitly that the motion of the Solar system was regular and that the solutions could be obtained as quasiperiodic series, using perturbation theory.

When Laskar $(1984,1985,1986)$ computed in an extensive way the secular equations for the Solar system, including all terms up to order 2 in the masses and 5 in eccentricity and inclination, he realized that the traditional perturbative methods could not be used for the integration of the secular equations, due to strong divergences that became apparent in the system of the inner planets (Laskar 1984). This difficulty was overcome by switching to a numerical integration of the secular equations, which could be done in a very effective way, with a very large stepsize of 500 years. These computations provided a much more accurate solution for the orbital motion of the Solar system (Laskar 1986, 1988), which also included a full solution for the precession and obliquity of the Earth for insolation computations over $10 \mathrm{Myr}$ (million of years). Extending his integration to $200 \mathrm{Myr}$, Laskar $(1989,1990)$ demonstrated that the orbital motion of the planets, and especially of the terrestrial planets, is chaotic, with an exponential divergence corresponding to an increase of the error by a factor of 10 every $10 \mathrm{Myr}$, thus destroying the hope to obtain a precise astronomical solution for paleoclimate studies over more than a few tens of million of years (Laskar 1999).

The first long term direct numerical integration (without averaging) of a realistic model of the Solar system, together with the precession and obliquity equations, was made by Quinn et al. (1991) over 3 Myr. Over its range, this solution presented very small differences with the updated secular solution of Laskar et al. (1993) that was computed over $20 \mathrm{Myr}$, and has since been extensively used for paleoclimate computations under the acronym La93. The orbital motion of the full Solar system has also been computed over $100 \mathrm{Myr}$ by Sussman \& Wisdom (1992), using a symplectic integrator with mixed variables (Wisdom \& Holman 1991), confirming the chaotic behaviour found by Laskar $(1989,1990)$. Following the improvement of computer technology, long term integrations of realistic models of the Solar system become more easy to perform, but are still challenging when the time interval is the age of the Solar system or if the accuracy of the model is comparable to the precision of short term planetary ephemeris. The longest integration was made over several billions of years by Ito \& Tanikawa (2002) with a Newtonian model that did not contain general relativity or lunar contributions, while a recent long term integration of the orbital motion of the Solar system including general relativity, and where the Moon is resolved was made by Varadi et al. (2003) over about 50 Myr.

Here we present the result of the efforts that we have conducted in the past years in our group in order to obtain a new solution for Earth paleoclimate studies over the Neogene pe$\operatorname{riod}(\approx 23 \mathrm{Myr})$ and beyond. After a detailed analysis of the main limiting factors for long term integrations (Laskar 1999), and the design of new symplectic integrators (Laskar \& Robutel 2001), we have obtained a new numerical solution for both orbital and rotational motion of the Earth that can be used over about 50 Myr for paleoclimate studies, and even over longer periods of time if only the most stable features of the solution are used. Apart from the use of a very complete dynamical model for the orbital motion of the planets, and of the new symplectic integrator of Laskar \& Robutel (2001) (see next section), a major change with respect to La93 (Laskar et al. 1993) is the consideration, in the precession solution, of a more complete model for the Earth-Moon tidal interactions (Sect. 4.1).

The present solution (La2004) or some of its previous variants have already been distributed (Laskar 2001) and used for calibration of sedimentary records over extended time interval (Pälike 2002), and in particular for the construction of a new astronomically calibrated geological time scale for the Neogene period (Lourens et al. 2004). The full solution is available on the Web site www.imcce.fr/ Equipes/ASD/insola/earth/earth.html, together with a set of routines for the computation of the insolation quantities following (Laskar et al. 1993).

In addition to the numerical output of the orbital and rotational parameter of the Earth that is available on the Web site, we have made a special effort to provide in Sect. 8 very compact analytical approximations for the orbital and rotational quantities of the Earth, that can be used in many cases for a better analytical understanding of the insolation variations. Finally, in the last sections, the stability of the solution La2004 is discussed, as well as the possible chaotic transitions of the arguments present in the main secular resonances.

\section{Numerical model}

The orbital solutions La90-93 (Laskar 1990; Laskar et al. 1993) were obtained by a numerical integration of the averaged equations of the Solar system, including the main general relativity and Lunar perturbations. The averaging process was performed using dedicated computer algebra routines. The resulting equations were huge, with about 150000 polynomial terms, but as the short period terms were no longer present, these equations could be integrated with a step size of 200 to 500 years, allowing very extensive long term orbital computations for the Solar system.

Although these averaged equations solutions could be improved by some new adjustment of the initial conditions and parameters (Laskar et al. 2004), it appears that because of the improvements of computer technology, it becomes now possible to obtain more precise results over a few tens of million of years using a more direct numerical integration of the gravitational equations, and this is how the present new 
Table 1. Main constants used in La2004. IAU76 refers to the resolutions of the International Astronmical Union of 1976, IERS1992, and IERS2000, refers to the IERS conventions (McCarthy 1992; McCarthy \& Petit 2004).

\begin{tabular}{|c|c|c|c|}
\hline Symbol & Value & Name & Ref. \\
\hline$\epsilon_{0}$ & 84381.448 & Obliquity of the ecliptic at J2000.0 & IAU76 \\
\hline$\omega_{0}$ & $7.292115 \times 10^{-5} \mathrm{rad} \mathrm{s}^{-1}$ & Mean angular velocity of the Earth at J2000.0 & IERS2000 \\
\hline$\psi_{0}$ & $5029.0966 " / c y$ & Precession constant & IERS2000 \\
\hline$\delta \psi_{0}$ & $-0.29965 " / \mathrm{cy}$ & Correction to the precession & IERS2000 \\
\hline$k 2$ & 0.305 & $k_{2}$ of the Earth & (Lambeck 1988) \\
\hline$k 2_{\mathrm{M}}$ & 0.0302 & $k_{2}$ of the Moon & (Yoder 1995) \\
\hline$\Delta t$ & $639 \mathrm{~s}$ & Time lag of the Earth & \\
\hline$\Delta t_{\mathrm{M}}$ & $7055 \mathrm{~s}$ & Time lag of the Moon & \\
\hline$J_{2 \mathrm{E}}$ & 0.0010826362 & Dynamical form-factor for the Earth & IERS1992 \\
\hline$J_{2 \mathrm{M}}$ & 0.000202151 & Dynamical form-factor for the Moon & IERS1992 \\
\hline$J_{2 \mathrm{~S}}$ & 2. $\times 10^{-7}$ & Dynamical form-factor for the Sun & IERS2000 \\
\hline$R_{\mathrm{E}}$ & $6378.1366 \mathrm{~km}$ & Equatorial radius of the Earth & IERS2000 \\
\hline$R_{\mathrm{M}}$ & $1738 \mathrm{~km}$ & Equatorial radius of the Moon & IAU76 \\
\hline$R_{\text {sun }}$ & $696000000 \mathrm{~m}$ & Equatorial radius of the Sun & IAU76 \\
\hline$G S$ & $1.32712442076 \times 10^{20} \mathrm{~m}^{3} \mathrm{~s}^{-2}$ & Heliocentric gravitational constant & IERS2000 \\
\hline$M_{\text {mer }}$ & 6023600 & Sun - Mercury mass ratio & IERS1992 \\
\hline$M_{\mathrm{ven}}$ & 408523.71 & Sun - Venus mass ratio & IERS1992 \\
\hline$M_{\text {ear }}$ & 328900.56 & Sun - Earth and Moon mass ratio & IERS1992 \\
\hline$M_{\text {mar }}$ & 3098708 & Sun - Mars mass ratio & IERS1992 \\
\hline$M_{\text {jup }}$ & 1047.3486 & Sun - Jupiter and satellites mass ratio & IERS1992 \\
\hline$M_{\text {sat }}$ & 3497.9 & Sun - Saturn and satellites mass ratio & IERS1992 \\
\hline$M_{\text {ura }}$ & 22902.94 & Sun - Uranus and satellites mass ratio & IERS1992 \\
\hline$M_{\text {nep }}$ & 19412.24 & Sun - Neptune and satellites mass ratio & IERS1992 \\
\hline$M_{\mathrm{plu}}$ & 135000000 & Sun - Pluto and Charon mass ratio & IERS1992 \\
\hline$\mu$ & 0.0123000383 & Moon-Earth mass ratio & IERS2000 \\
\hline
\end{tabular}

solution La2004 is obtained. A detailed account of the dynamical equations and numerical integrator is not in the scope of the present paper, but will be made in a forthcoming publication. Nevertheless, we will report here on the main issues concerning the numerical integrator, and will more extensively discuss on the dissipative terms in the integration (see Sect. 4.1).

\subsection{Dynamical model}

The orbital model differs from La93, as it comprises now all 9 planets of the Solar System, including Pluto. The postNewtonian general relativity corrections of order $1 / c^{2}$ due to the Sun are included following Saha \& Tremaine (1994).

The Moon is treated as a separate object. In order to obtain a realistic evolution of the Earth-Moon system, we also take into account the most important coefficient $\left(J_{2}^{\mathrm{S}}\right)$ in the gravitational potential of the Earth and of the Moon (Table 1), and the tidal dissipation in the Earth-Moon System (see Sect. 4.1). We also integrate at the same time the precession and obliquity equations for the Earth and the evolution of its rotation period in a comprehensive and coherent way, following the lines of Néron de Surgy \& Laskar (1997), Correia et al. (2003) (see Sect. 3).

\subsection{Numerical integrator}

In order to minimize the accumulation of roundoff errors, the numerical integration was performed with the new symplectic integrator scheme $S A B A C_{4}$ of (Laskar \& Robutel 2001), with a correction step for the integration of the Moon. This integrator is particularly adapted to perturbed systems where the Hamiltonian governing the equations of motion can be written in the form $H=A+\varepsilon B$, as the sum of an integrable part $A$ (the Keplerian equations of the planets orbiting the Sun and of the Moon around the Earth), and a small perturbation potential $\varepsilon B$ (here the small parameter $\epsilon$ is of the order of the planetary masses). Using this integrator with step size $\tau$ is then equivalent to integrate exactly a close by Hamiltonian $\tilde{H}$, where the error of method $H-\tilde{H}$ is of the order of $O\left(\tau^{8} \epsilon\right)+O\left(\tau^{2} \epsilon^{2}\right)$, and even $O\left(\tau^{8} \epsilon\right)+O\left(\tau^{4} \epsilon^{2}\right)$ when the correction step is added, 
while the same quantity is of the order $O\left(\tau^{2} \epsilon\right)$, in the widely used symplectic integrator of (Wisdom \& Holman 1991). More precisely, the integration step $S_{0}(\tau)$ with $S A B A_{4}$ is

$$
\begin{aligned}
S_{0}(\tau)= & \mathrm{e}^{\tau c_{1} L_{A}} \mathrm{e}^{\tau d_{1} L_{B}} \mathrm{e}^{\tau c_{C} L_{A}} \mathrm{e}^{\tau d_{2} L_{B}} \mathrm{e}^{\tau c_{3} L_{A}} \\
& \times \mathrm{e}^{\tau d_{2} L_{B}} \mathrm{e}^{\tau c_{2} L_{A}} \mathrm{e}^{\tau d_{1} L_{B}} \mathrm{e}^{\tau c_{1} L_{A}}
\end{aligned}
$$

where $\tau$ is the integration step, $L_{f}$ is the differential operator defined by $L_{f} g=\{f, g\}$, where $\{f, g\}$ is the usual Poisson bracket, and the coefficients $c_{i}$ and $d_{i}$ are

$$
\begin{aligned}
& c_{1}=1 / 2-\sqrt{525+70 \sqrt{30}} / 70 \\
& c_{2}=(\sqrt{525+70 \sqrt{30}}-\sqrt{525-70 \sqrt{30}}) / 70 \\
& c_{3}=(\sqrt{525-70 \sqrt{30}}) / 35 \\
& d_{1}=1 / 4-\sqrt{30} / 72 \\
& d_{2}=1 / 4+\sqrt{30} / 72 .
\end{aligned}
$$

For the integration of the Earth-Moon system, we have added a correction step (see Laskar \& Robutel 2001), and the full integrator steps $S_{1}(\tau)$ of $S A B A C_{4}$ then becomes

$S_{1}(\tau)=\mathrm{e}^{-\tau^{3} \varepsilon^{2} b / 2 L_{C}} S_{0}(\tau) \mathrm{e}^{-\tau^{3} \varepsilon^{2} b / 2 L_{C}}$

where $b=0.00339677504820860133$ and $C=\{\{A, B\}, B\}$. The step size used in the integration was $\tau=5 \times 10^{-3} \mathrm{yr}=$ 1.82625 days. The initial conditions of the integration were least square adjusted to the JPL ephemeris DE406, in order to compensate for small differences in the model. In particular, we do not take into account the effect of the minor planets, and the modelization of the body interactions in the Earth-Moon system is more complete in DE406 (see Williams et al. 2001). The integration time for our complete model, with $\tau=5 \times 10^{-3} \mathrm{yr}$ is about one day per 5 Myr on a Compaq Alpha (ev68, 833 Mhz) workstation.

\subsection{Numerical roundoff error}

In Fig. 1a is plotted the evolution of the total energy of the system from $-250 \mathrm{Myr}$ to $+250 \mathrm{Myr}$. The energy presents a secular trend that corresponds to the dissipation in the Earth-Moon system. Indeed, after removing the computed energy change due to the secular evolution of the Earth-Moon semi-major axis, the secular trend in the energy evolution disappears and we are left with a residual that is smaller than $2.5 \times 10^{-10}$ after $250 \mathrm{Myr}$, and seems to behave as a random walk (Fig. 1b). The normal component of the angular momentum is conserved over the same time with a relative error of less than $1.3 \times 10^{-10}$ (Fig. 1c).

In order to test the randomness of the numerical error in the integration, we have plotted the distribution of the difference of total energy from one 1000 year step to the next. After normalization to 1 of the area of the distribution curve (Fig. 2), we obtain a curve that is almost identical to the normalized Gaussian function

$$
f(t)=\frac{1}{\sigma \sqrt{2 \pi}} \exp \left(-\frac{x^{2}}{2 \sigma^{2}}\right)
$$

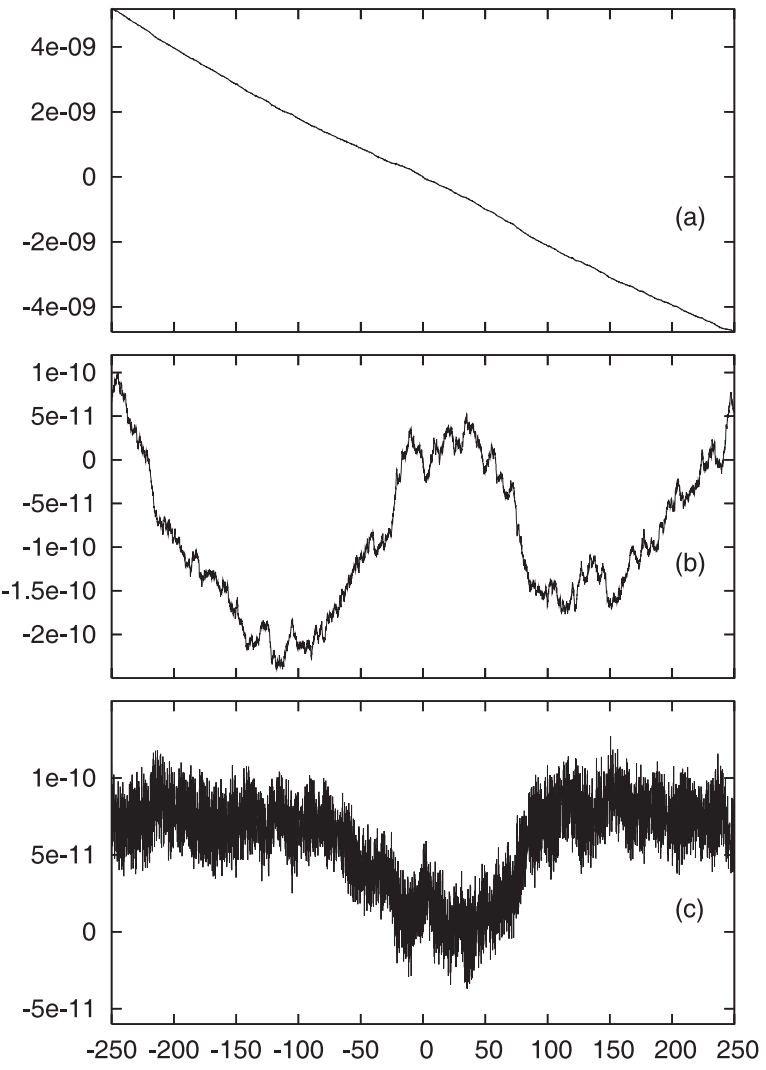

Fig. 1. Conservation of integrals. a) Relative variation of the total energy of the system versus time (in Myr) from $-250 \mathrm{Myr}$ to $+250 \mathrm{Myr}$. b) The same after correction of the secular trend due to the tidal dissipation in the Earth-Moon system. c) Relative variation of the normal component of the total angular momentum.

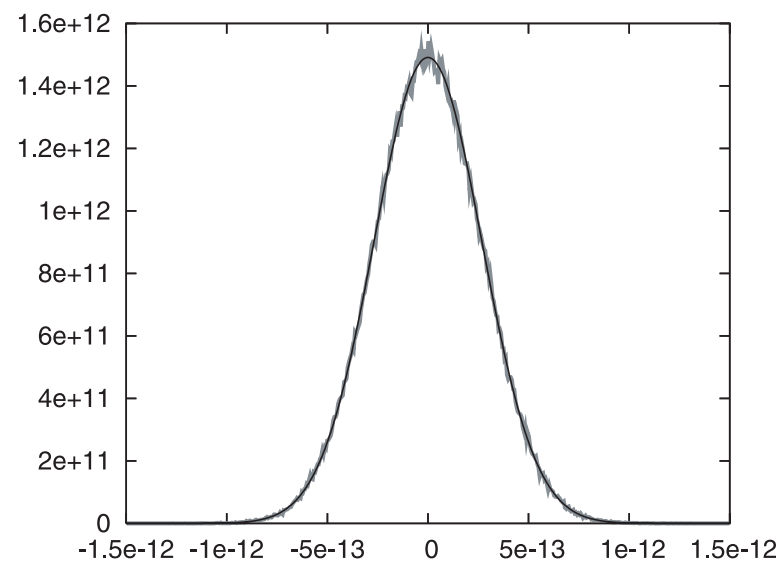

Fig. 2. Normalized repartition of the energy numerical error for $1000 \mathrm{yr}$ steps, over the whole integration, from $-250 \mathrm{Myr}$ to +250 Myr. Practically superposed to this curve is the computed normal distribution given by Eqs. (4) and (5).

where $\sigma=2.6756 \times 10^{-13}$ is the computed standard deviation

$\sigma=\sqrt{\sum_{i}\left(x_{i}-m\right)^{2}} /(N-1)$

while the mean $m=-2.6413 \times 10^{-18}$ is neglected. The integration stepsize is $5 \times 10^{-3} \mathrm{yr}$, the standard deviation per step is thus $\sigma_{1}=5.9828 \times 10^{-16}$, that is about $2.7 \varepsilon_{\mathrm{M}}$, 


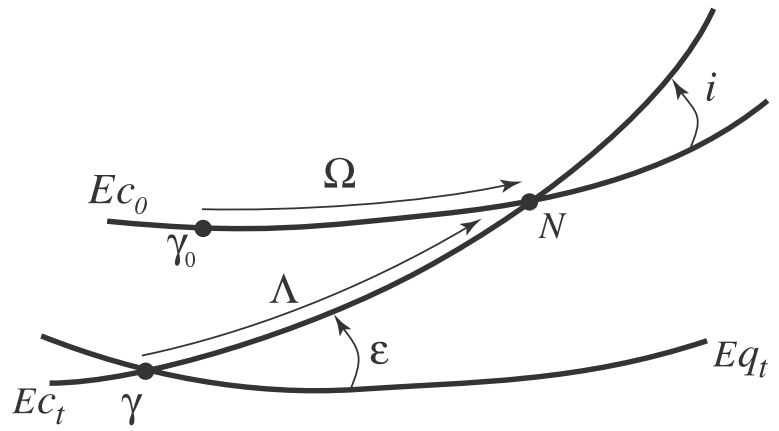

Fig. 3. Fundamental planes for the definition of precession and obliquity. $E_{q_{t}}$ and $E_{c_{t}}$ are the mean equator and ecliptic at date $t . E_{c_{0}}$ is the fixed ecliptic at Julian date J2000, with equinox $\gamma_{0}$. The general precession in longitude $\psi$ is defined by $\psi=\Lambda-\Omega . \omega$ is the longitude of the node, and $i$ the inclination. The angle $\varepsilon$ between $E_{q_{t}}$ and $E_{c_{t}}$ is the obliquity.

where $\varepsilon_{\mathrm{M}} \approx 2.22 \times 10^{-16}$ is the machine Epsilon in double precision. Assuming that the machine error is uniformly distributed in the interval $\left[-\varepsilon_{\mathrm{M}} / 2,+\varepsilon_{\mathrm{M}} / 2\right]$, the standard error of an elementary operation is $\sigma_{0}=\varepsilon_{\mathrm{M}} / \sqrt{12}$, and the error over one step thus corresponds to $\left(\sigma_{1} / \sigma_{0}\right)^{2} \approx 87$ elementary operations. This value is thus extremely small, and we believe that we will not be able to improve $\sigma_{1}$ unless we decrease $\sigma_{0}$ by switching to higher machine precision. For example, in quadruple precision (128 bits for the representation of a real number), $\varepsilon_{M} \approx 1.93 \times 10^{-34}$. Moreover, the Gaussian distribution of the increments of the energy (Fig. 2) ensures that we do not have systematic trends in our computations, and that the numerical errors behave as a random variable with zero mean. The total energy error reflects mostly the behavior of the outer planets, but we can assume that the numerical error will behave in roughly the same for all the planets, although for Mercury, due to its large eccentricity, some slight increase of the error may occur. This should not be the case for the error of method, resulting from the truncation in power of $\tau$ in the integrator, where the error should decrease as the period of the planet increases.

\section{Precession equations}

We suppose here that the Earth is a homogeneous rigid body with moments of inertia $A<B<C$ and we assume that its spin axis is also the principal axis of inertia. The precession $\psi$ and obliquity $\varepsilon$ (Fig. 3) equations for the rigid Earth in the presence of planetary perturbations are given by Kinoshita (1977), Laskar (1986), Laskar et al. (1993), Néron de Surgy \& Laskar (1997).

$$
\left\{\begin{array}{l}
\frac{\mathrm{d} X}{\mathrm{~d} t}=L \sqrt{1-\frac{X^{2}}{L^{2}}}(\mathcal{B}(t) \sin \psi-\mathcal{A}(t) \cos \psi) \\
\frac{\mathrm{d} \psi}{\mathrm{d} t}=\frac{\alpha X}{L}-\frac{X}{L \sqrt{1-\frac{X^{2}}{L^{2}}}}(\mathcal{A}(t) \sin \psi+\mathcal{B}(t) \cos \psi)-2 C(t) .
\end{array}\right.
$$

With $X=\cos \varepsilon, L=C \omega$, where $\omega$ is the spin rate of the Earth, and

$$
\left\{\begin{array}{l}
\mathcal{A}(t)=\frac{2}{\sqrt{1-p^{2}-q^{2}}}[\dot{q}+p(q \dot{p}-p \dot{q})] \\
\mathcal{B}(t)=\frac{2}{\sqrt{1-p^{2}-q^{2}}}[\dot{p}-q(q \dot{p}-p \dot{q})] \\
C(t)=q \dot{p}-p \dot{q}
\end{array}\right.
$$

where $q=\sin (i / 2) \cos \Omega$ and $p=\sin (i / 2) \sin \Omega$, and where $\alpha$ is the "precession constant":

$$
\begin{aligned}
\alpha= & \frac{3 G}{2 \omega}\left[\frac{m_{\odot}}{\left(a_{\odot} \sqrt{1-e_{\odot}^{2}}\right)^{3}}\right. \\
& \left.+\frac{m_{\mathrm{M}}}{\left(a_{\mathrm{M}} \sqrt{1-e_{\mathrm{M}}^{2}}\right)^{3}}\left(1-\frac{3}{2} \sin ^{2} i_{\mathrm{M}}\right)\right] E_{\mathrm{d}} .
\end{aligned}
$$

For a fast rotating planet like the Earth, the dynamical ellipticity $E_{\mathrm{d}}=(2 C-A-B) / C$ can be considered as proportional to $\omega^{2}$; this corresponds to the hydrostatic equilibrium (see for example Lambeck 1980). In this approximation, $\alpha$ is thus proportional to $\omega$. The quantities $\mathcal{A}, \mathcal{B}$ and $C$ are related to the secular evolution of the orbital plane of the Earth and are given by the integration of the planetary motions.

\section{Contributions of dissipative effects}

\subsection{The body tides}

\subsubsection{Rotational evolution}

Following (Darwin 1880; Mignard 1979), we assume that the torque resulting from tidal friction is proportional to the time lag $\Delta t$ needed for the deformation to reach the equilibrium. This time lag is supposed to be constant, and the angle between the direction of the tide-raising body and the direction of the high tide (which is carried out of the former by the rotation of the Earth) is proportional to the speed of rotation. Such a model is called "viscous", and corresponds to the case for which $1 / Q$ is proportional to the tidal frequency.

After averaging over the mean anomaly of the Moon and Earth (indices $M$ and $\oplus$ ), and over the longitude of node and perigee of the Moon, we have at second order in eccentricity for the contributions of the solar tides

$$
\left\{\begin{aligned}
\frac{\mathrm{d} L}{\mathrm{~d} t}= & -\frac{3 G m_{\odot}^{2} R^{5} k_{2} \Delta t}{2 a_{\odot}{ }^{6}} \\
& \times\left[\left(1+\frac{15}{2} e_{\odot}^{2}\right)\left(1+\frac{X^{2}}{L^{2}}\right) \frac{L}{C}-2\left(1+\frac{27}{2} e_{\odot}^{2}\right) \frac{X}{L} n_{\odot}\right] \\
\frac{\mathrm{d} X}{\mathrm{~d} t}= & -\frac{3 G m_{\odot}^{2} R^{5} k_{2} \Delta t}{2 a_{\odot}^{6}} \\
& \times\left[2\left(1+\frac{15}{2} e_{\odot}^{2}\right) \frac{X}{C}-2\left(1+\frac{27}{2} e_{\odot}^{2}\right) n_{\odot}\right]
\end{aligned}\right.
$$


where $n_{\odot}$ is the mean motion of the Sun (index $\odot$ ) around the Earth, while the contribution of the lunar tides is

$$
\left\{\begin{array}{l}
\frac{\mathrm{d} L}{\mathrm{~d} t}=-\frac{3 G m_{\mathrm{M}}^{2} R^{5} k_{2} \Delta t}{2 a_{\mathrm{M}}^{6}} \\
\times\left[\frac{1}{2}\left(1+\frac{15}{2} e_{\mathrm{M}}^{2}\right)\left[3-\cos ^{2} i_{\mathrm{M}}+\left(3 \cos ^{2} i_{\mathrm{M}}-1\right) \frac{X^{2}}{L^{2}}\right] \frac{L}{C}\right. \\
\left.-2\left(1+\frac{27}{2} e_{\mathrm{M}}^{2}\right) \frac{X}{L} n_{\mathrm{M}} \cos i_{\mathrm{M}}\right] \\
\frac{\mathrm{d} X}{\mathrm{~d} t}=-\frac{3 G m_{\mathrm{M}}^{2} R^{5} k_{2} \Delta t}{2 a_{\mathrm{M}}^{6}} \\
\times\left[\left(1+\frac{15}{2} e_{\mathrm{M}}^{2}\right)\left(1+\cos ^{2} i_{\mathrm{M}}\right) \frac{X}{C}\right. \\
\left.-2\left(1+\frac{27}{2} e_{\mathrm{M}}^{2}\right) n_{\mathrm{M}} \cos i_{\mathrm{M}}\right] .
\end{array}\right.
$$

The "cross tides", are obtained by the perturbation of the tidal bulge raised by the Sun or the Moon on the other body. Their importance was noticed by Touma \& Wisdom (1994). These tidal effects tend to drive the equator towards the ecliptic, and have both the same magnitude

$$
\left\{\begin{array}{l}
\frac{\mathrm{d} L}{\mathrm{~d} t}=-\frac{3 G m_{\odot} m_{\mathrm{M}} R^{5} k_{2} \Delta t}{4 a_{\odot}{ }^{3} a_{\mathrm{M}}{ }^{3}} \\
\times\left(1+\frac{3}{2} e_{\odot}^{2}\right)\left(1+\frac{3}{2} e_{\mathrm{M}}^{2}\right)\left(3 \cos ^{2} i_{\mathrm{M}}-1\right)\left(1-\frac{X^{2}}{L^{2}}\right) \frac{L}{C} \\
\frac{\mathrm{d} X}{\mathrm{~d} t}=0 .
\end{array}\right.
$$

\subsubsection{Orbital evolution}

At second order in eccentricity, we have for the orbital evolution of the Moon (Mignard 1979, 1980, 1981; Néron de Surgy \& Laskar 1997)

$$
\left\{\begin{aligned}
& \frac{\mathrm{d} a_{\mathrm{M}}}{\mathrm{d} t}= \frac{6 G m_{\mathrm{M}}^{2} R^{5} k_{2} \Delta t}{\mu a_{\mathrm{M}}^{7}} \\
& \times\left[\left(1+\frac{27}{2} e_{\mathrm{M}}^{2}\right) \frac{X}{C n_{\mathrm{M}}} \cos i_{\mathrm{M}}-\left(1+23 e_{\mathrm{M}}^{2}\right)\right] \\
& \frac{\mathrm{d} e_{\mathrm{M}}}{\mathrm{d} t}= \frac{3 G m_{\mathrm{M}}^{2} R^{5} k_{2} \Delta t e_{\mathrm{M}}}{\mu a_{\mathrm{M}}^{8}}\left[\frac{11}{2} \frac{X}{C n_{\mathrm{M}}} \cos i_{\mathrm{M}}-9\right] \\
& \frac{\mathrm{d} \cos i_{\mathrm{M}}}{\mathrm{d} t}=\frac{3 G m_{\mathrm{M}}^{2} R^{5} k_{2} \Delta t}{2 \mu a_{\mathrm{M}}{ }^{8}}\left(1+8 e_{\mathrm{M}}^{2}\right) \frac{X}{C n_{\mathrm{M}}} \sin ^{2} i_{\mathrm{M}}
\end{aligned}\right.
$$

where $\mu=m_{\oplus} m_{\mathrm{M}} /\left(m_{\oplus}+m_{\mathrm{M}}\right)$, and for the Sun

$$
\left\{\begin{aligned}
\frac{\mathrm{d} a_{\odot}}{\mathrm{d} t}= & \frac{6 G m_{\odot}^{2} R^{5} k_{2} \Delta t}{\mu^{\prime} a_{\odot}^{7}} \\
& \times\left[\left(1+\frac{27}{2} e_{\odot}^{2}\right) \frac{X}{C n_{\odot}}-\left(1+23 e_{\odot}^{2}\right)\right] \\
\frac{\mathrm{d} e_{\odot}}{\mathrm{d} t}= & \frac{3 G m_{\odot}^{2} R^{5} k_{2} \Delta t e_{\odot}}{\mu^{\prime} a_{\odot}^{8}}\left[\frac{11}{2} \frac{X}{C n_{\odot}}-9\right]
\end{aligned}\right.
$$

where $\mu^{\prime}=m_{\oplus} m_{\odot} /\left(m_{\oplus}+m_{\odot}\right)$. However, both last variations are negligible: about 3 meters per Myr for $\mathrm{d} a_{\odot} / \mathrm{d} t$ and $10^{-12}$ per Myr for $\mathrm{d} e_{\odot} / \mathrm{d} t$.

\subsubsection{Tides on the Moon}

We have also taken into account the tidal effect raised by the Earth on the Moon, following Néron de Surgy \& Laskar (1997), with the following simplifying assumptions that the Moon is locked in synchronous spin-orbit resonance with the Earth (i.e. $\omega_{\mathrm{M}}=n_{\mathrm{M}}$ ), and expanding only at first order in the Moon inclination $\left(6.41^{\circ}\right.$ at $\left.t=\mathrm{J} 2000\right)$. We thus obtain the additional contributions

$\left\{\begin{array}{l}\frac{\mathrm{d} a_{\mathrm{M}}}{\mathrm{d} t}=-\frac{57 G m_{\oplus}^{2} R_{\mathrm{M}}^{5} k_{2 \mathrm{M}} \Delta t_{\mathrm{M}} e_{\mathrm{M}}^{2}}{\mu a_{\mathrm{M}}^{7}} \\ \frac{\mathrm{d} e_{\mathrm{M}}}{\mathrm{d} t}=-\frac{21 G m_{\oplus}^{2} R_{\mathrm{M}}^{5} k_{2 \mathrm{M}} \Delta t_{\mathrm{M}} e_{\mathrm{M}}}{2 \mu a_{\mathrm{M}}^{8}} .\end{array}\right.$

With the numerical values from Table 1 , these contributions represent $1.2 \%$ of the total $\mathrm{d} a_{\mathrm{M}} / \mathrm{d} t$ and $30 \%$ of the total $\mathrm{d} e_{\mathrm{M}} / \mathrm{d} t$ for present conditions. Finally, the tides raised on the Moon by the Sun can be neglected because the ratio of the magnitudes of solar and terrestrial tides on the Moon is

$\left(\frac{m_{\odot}}{m_{\oplus}}\right)^{2}\left(\frac{a_{\odot}}{a_{\mathrm{M}}}\right)^{6} \simeq 3.2 \times 10^{-5}$

\subsection{Other dissipative effects}

\subsubsection{Core-mantle friction}

The core and the mantle have different dynamical ellipticities, so they tend to have different precession rates. This trend produces a viscous friction at the core-mantle boundary (Poincaré 1910; Rochester 1976; Lumb \& Aldridge 1991). We will consider here that an effective viscosity $v$ can account for a weak laminar friction, as well as a strong turbulent one which thickens the boundary layer (e.g. Correia et al. 2003).

The core-mantle friction tends to slow down the rotation and to bring the obliquity down to $0^{\circ}$ if $\varepsilon<90^{\circ}$ and up to $180^{\circ}$ otherwise, which contrasts with the effect of the tides. Furthermore, one can see that, despite the strong coupling by pressure forces, there can be a substantial contribution to the variation of the spin for high viscosities and moderate speeds of rotation.

\subsubsection{Atmospheric tides}

The Earth's atmosphere also undergoes some torques which can be transmitted to the surface by friction: a torque caused by the gravitational tides raised by the Moon and the Sun, a magnetic one generated by interactions between the magnetosphere and the solar wind. Both effects are negligible; see respectively Chapman \& Lindzen (1970) and Volland (1978).

Finally, a torque is produced by the daily solar heating which induces a redistribution of the air pressure, mainly driven by a semidiurnal wave, hence the so-called thermal atmospheric tides (Chapman \& Lindzen 1970). The axis of symmetry of the resulting bulge of mass is permanently shifted out of the direction of the Sun by the Earth's rotation. As for the body tides, this loss of symmetry is responsible for the torque which, in the present conditions, tends to accelerate the spin (Correia \& Laskar 2003). 
Volland (1978) showed that this effect is not negligible, reducing the Earth's despinning by about 7.5\%. But, although the estimate of their long term contributions deserves careful attention, we have not taken the atmospheric tides into account in the computations presented in the next sections, assuming that, as the uncertainty on some of the other factors is still large, the global results obtained here will not differ much when taking this additional effect into consideration.

\subsubsection{Mantle convection}

Several internal geophysical processes are able to affect the moments of inertia of the Earth and thereby its precession constant value. Redistribution of mass within the Earth can occur as a consequence of plate subduction, upwelling plumes, mantle avalanches or density anomalies driven by mantle convection. Typical time-scales of these phenomena range from $\sim 10$ to 100 Myr. However, their impact is still poorly known. Forte \& Mitrovica (1997) computed convection-induced perturbations in the dynamical ellipticity of the Earth over the last $20 \mathrm{Myr}$, using different sets of 3D seismic heterogeneity models and mantle viscosity profiles. Most of the models indicate that mantle convection led to a mean relative increase of the precession constant close to $\sim 0.5 \%$ since the beginning of the Neogene period ( $23 \mathrm{Myr}$ ago). Although this value is similar to our estimate of the tidal dissipation effect (but in the opposite direction, see Sect. 9), we did not take account of its impact in our computations. Because of its still large uncertainty, we feel that it is more appropriate to include its possible contribution as a perturbation of the tidal dissipation parameter.

\subsubsection{Climate friction}

Climate friction is a positive and dissipative feedback between obliquity variations and climate which may cause a secular drift of the spin axis. In response to quasi-periodic variations in the obliquity, glacial and interglacial conditions drive transport of water into and out of the polar regions, affecting the dynamical ellipticity of the Earth. A significant fraction of the surface loading is compensated by viscous flow within the Earth, but delayed responses in both climatic and viscous relaxation processes may introduce a secular term in the obliquity evolution (Rubincam 1990, 1995). Because both the ice load history and the visco-elastic structure of the Earth are not strongly constrained, it is difficult to produce accurate simulations and predictions of the coupled response of the entire system. Nevertheless, simplifying assumptions have been used to estimate the magnitude and the direction of the secular drift. Several analyses have examined this phenomenon, suggesting that climate friction could have changed the Earth's obliquity by more that $20^{\circ}$ over its whole geological history (Bills 1994; Ito et al. 1995; Williams et al. 1998). A more detailed theoretical and numerical treatment of climate friction has been developped by Levrard \& Laskar (2003). Using available constraints on the ice volume response to obliquity forcing based on $\delta^{18} \mathrm{O}$ oxygen-isotope records, they showed that climate friction impact is likely negligible. Over the last $3 \mathrm{Ma}$,

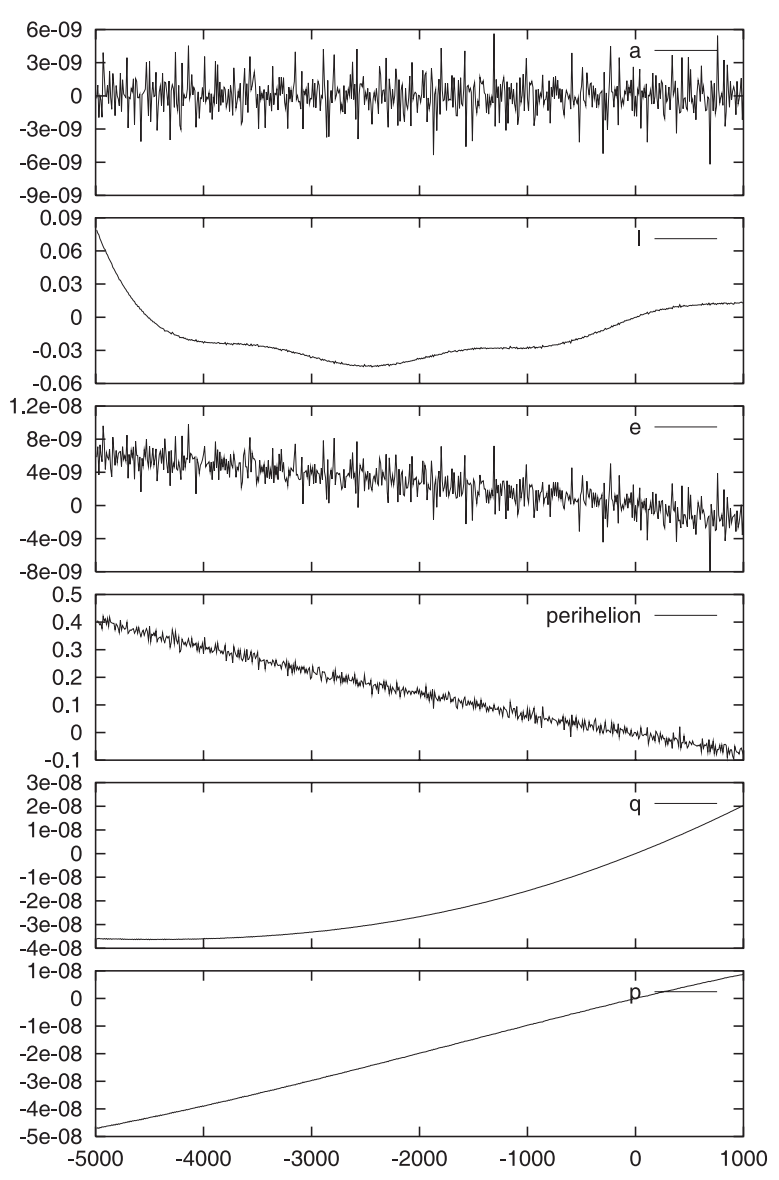

Fig. 4. Differences La2003-DE406 over the full range of DE406 $(-5000 \mathrm{yr}$ to +1000 from $\mathrm{J} 2000)$. The units for semi-major axis $(a)$ are $\mathrm{AU}$, arcsec for mean longitude $(l)$ and longitude of perihelion (perihelion). The eccentricity $(e)$ and the inclinations variables $(q=$ $\sin (i / 2) \cos (\Omega), p=\sin (i / 2) \sin (\Omega)$ ), where $i$ and $\Omega$ are the inclination and node from the ecliptic and equinox J2000.

corresponding to the intensification of the Northern Hemisphere glaciation, a maximal absolute drift of only $\sim 0.04^{\circ} / \mathrm{Myr}$ has been estimated for a realistic lower mantle viscosity of $10^{22} \mathrm{~Pa}$. s. Before $3 \mathrm{Ma}$, climate friction impact is expected to be much more negligible because of the lack of massive ice caps. In this context, this effect was not taken into account in our long-term computations. Indeed, the uncertainty on the tidal dissipation is still important and it is expected that most of the possible effect of climate friction would be absorbed by a small change of the main tidal dissipation parameters.

Nevertheless, glaciation-induced perturbations in the dynamical ellipticity of the Earth change the precession frequency and thereby the obliquity frequencies leading to a temporal shift between a perturbated and a nominal solution (see Sect. 9). Estimates of this offset have been performed by Mitrovica \& Forte (1995) and Levrard \& Laskar (2003) for a large set of mantle viscosity profiles. It may reach between $\sim 0.7$ and $8 \mathrm{kyr}$ after $3 \mathrm{Myr}$, corresponding respectively to extreme lower mantle viscosities of $3 \times 10^{21}$ and $10^{23} \mathrm{~Pa}$. s. In the last case, the Earth is nearly rigid on orbital timescales and the corresponding offset can be thus reasonably considered as a maximal value. 


\section{Comparison with DE406}

Using a direct numerical integrator, our goal is to provide a long term solution for the orbital and precessional elements of the Earth with a precision that is comparable with the usual accuracy of a short time ephemeris. We have thus compared our solution with the most advanced present numerical integration, DE406, that was itself adjusted to the observations (Standish 1998). Over the full range of DE406, that is from $-5000 \mathrm{yr}$ to $+1000 \mathrm{yr}$ from the present date, the maximum difference in the position of the Earth-Moon barycenter is less than 0.09 arcsec in longitude, and the difference in eccentricity less than $10^{-8}$. The difference in the longitude of the Moon are more important, as the dissipative models that are used in the two integrations are slightly different. They amount to 240 arcsec after 5000 years, and the eccentricity difference is of the order of $2 \times 10^{-5}$, but it should be noted that only the averaged motion of the Moon will have some significant influence on the precession and obliquity of the Earth. Table 2 summarizes the maximum differences between La2004 and DE406 for the main orbital elements, over the whole interval of DE406, for different time intervals.

\section{Comparison with La93}

We have compared in Figs. 5 and 6 the eccentricity and inclination of the secular solution La93 and the new present solution La2004. Over $10 \mathrm{Myr}$, the two orbital solutions La93 and La2004 are nearly identical, and do not differ significantly over 20 Myr. The differences in eccentricity increase regularly with time, amounting to about 0.02 in the eccentricity after $20 \mathrm{Myr}$, about $1 / 3$ of the total amplitude $\approx 0.063$, while the difference in the orbital inclination reaches 1 degree, to compare to a maximum variation of 4.3 degrees. These differences result mostly from a small difference in the main secular frequency $g_{6}$ from Jupiter and Saturn that is now $g_{6}=28.2450 \mathrm{arcsec} / \mathrm{year}$ (Table 3) instead of 28.2207 arcsec/year in the previous solution. Beyond 20 million years, the differences between the two solutions become more noticeable. The solutions in obliquity and climatic precession are plotted in Figs. 7 and 8. The difference in obliquity over $20 \mathrm{Myr}$ amounts to about 2 degrees, which means that the obliquity cycles of the two solutions become nearly out of phase after this date. The differences La2004-La93 are plotted in (a), while in (b) only the precession model has been changed and in (c) only the orbital model has been changed. Comparing $7 \mathrm{~b}$ and $7 \mathrm{c}$ shows that most of the differences between the La93 and La2004 obliquity solutions result from the change in the dissipative model of the Earth-Moon system, while the only change of the orbital solution would lead to a much smaller change of only 0.6 degree in obliquity after $20 \mathrm{Myr}$. Similar conclusions can be made for the climatic precession (Figs. 8a-c).

\section{Secular frequencies}

Over long time scales, the determination of longitude of the planet on its orbit is not very important, and we are more concerned by the slow evolution of the Earth orbit under secular planetary perturbations. The semi-major axis of the Earth
Table 2. Maximum difference between La2004 and DE406 over the whole time interval of DE406 $(-5000 \mathrm{yr}$ to $+1000 \mathrm{yr}$ with origin at J2000); Col. 1: -100 to $+100 \mathrm{yr}$; Col. $2:-1000$ to $+1000 \mathrm{yr}$; Col. $3:-5000$ to +1000 yr. EMB is the Earth-Moon barycenter.

\begin{tabular}{|c|c|c|c|}
\hline & \multicolumn{3}{|c|}{$\lambda\left({ }^{\prime \prime} \times 1000\right)$} \\
\hline Mercury & 8 & 60 & 90 \\
\hline Venus & 12 & 107 & 143 \\
\hline EMB & 3 & 28 & 79 \\
\hline Mars & 19 & 171 & 277 \\
\hline Jupiter & 5 & 69 & 69 \\
\hline Saturn & 1 & 4 & 7 \\
\hline Uranus & 1 & 3 & 4 \\
\hline Neptune & 2 & 7 & 16 \\
\hline Pluto & 3 & 9 & 17 \\
\hline Moon & 13470 & 85873 & 221724 \\
\hline \multirow[t]{2}{*}{ Earth } & 10 & 88 & 238 \\
\hline & \multicolumn{3}{|c|}{$a\left(\mathrm{UA} \times 10^{10}\right)$} \\
\hline Mercury & 4 & 43 & 58 \\
\hline Venus & 13 & 23 & 27 \\
\hline EMB & 29 & 62 & 62 \\
\hline Mars & 27 & 76 & 76 \\
\hline Jupiter & 268 & 470 & 565 \\
\hline Saturn & 743 & 1073 & 1168 \\
\hline Uranus & 1608 & 3379 & 3552 \\
\hline Neptune & 3315 & 5786 & 6458 \\
\hline Pluto & 4251 & 10603 & 10603 \\
\hline Moon & 23 & 204 & 520 \\
\hline \multirow[t]{2}{*}{ Earth } & 487 & 3918 & 10238 \\
\hline & \multicolumn{3}{|c|}{$e\left(\times 10^{10}\right)$} \\
\hline Mercury & 33 & 52 & 144 \\
\hline Venus & 12 & 33 & 103 \\
\hline EMB & 30 & 80 & 98 \\
\hline Mars & 62 & 130 & 468 \\
\hline Jupiter & 30 & 95 & 158 \\
\hline Saturn & 69 & 107 & 138 \\
\hline Uranus & 99 & 135 & 144 \\
\hline Neptune & 65 & 177 & 201 \\
\hline Pluto & 92 & 215 & 215 \\
\hline Moon & 11152 & 252832 & 252832 \\
\hline \multirow[t]{2}{*}{ Earth } & 460 & 3669 & 8694 \\
\hline & \multicolumn{3}{|c|}{$\sin (i / 2)\left(\times 10^{10}\right)$} \\
\hline Mercury & 2 & 18 & 88 \\
\hline Venus & 5 & 55 & 144 \\
\hline EMB & 17 & 190 & 414 \\
\hline Mars & 48 & 492 & 2053 \\
\hline Jupiter & 1 & 11 & 122 \\
\hline Saturn & 1 & 6 & 100 \\
\hline Uranus & 2 & 5 & 5 \\
\hline Neptune & 2 & 6 & 6 \\
\hline Pluto & 5 & 13 & 17 \\
\hline Moon & 5765 & 97275 & 600789 \\
\hline Earth & 19 & 202 & 841 \\
\hline
\end{tabular}



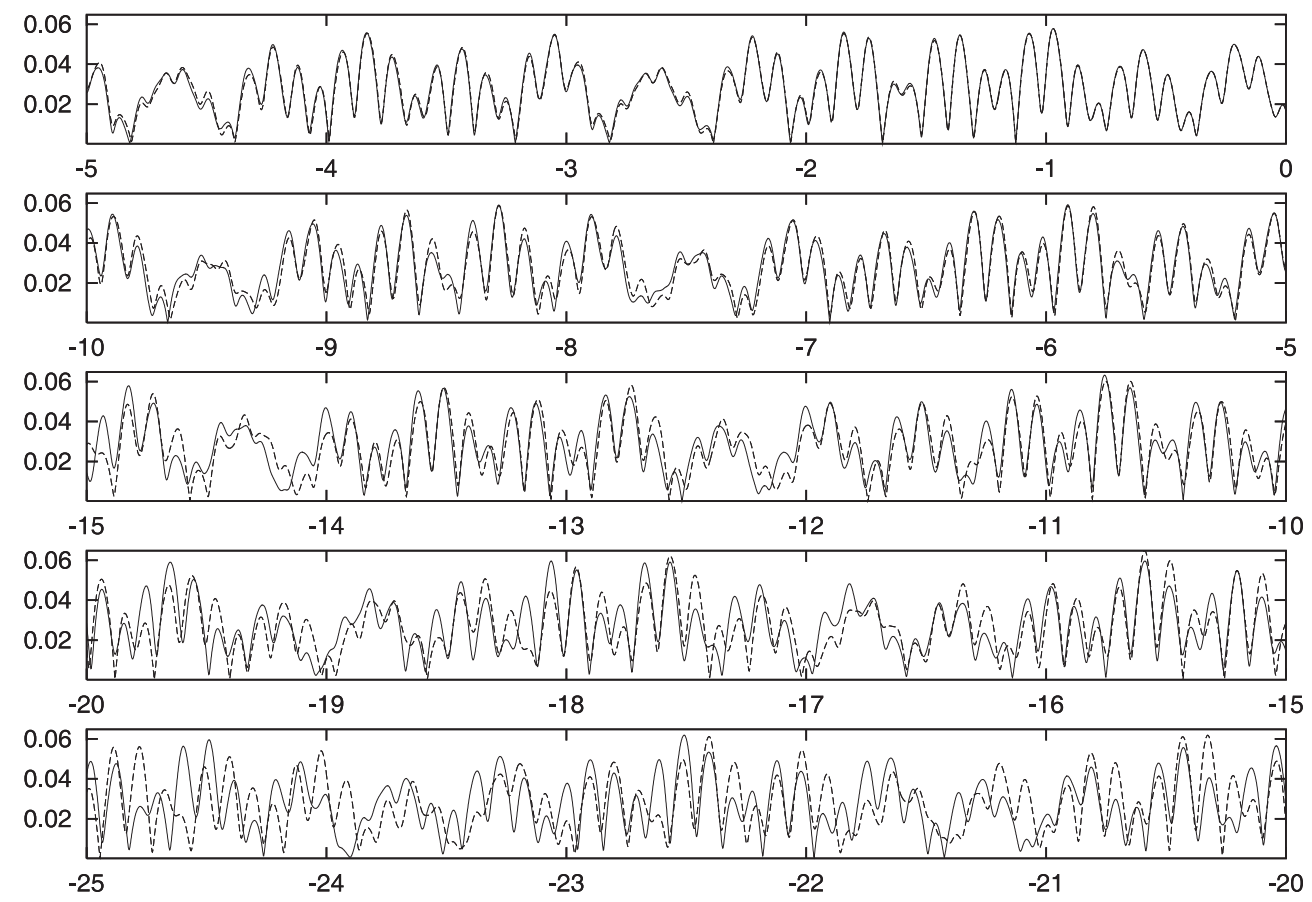

Fig. 5. Eccentricity of the Earth over 25 Myr in negative time from J2000. The solid line stands for the present solution La2004, while the dotted line is the eccentricity in the La93 solution (Laskar et al. 1993). The differences of the two solutions becomes noticeable after 10 Myr, and significantly different after 15-20 Myr.

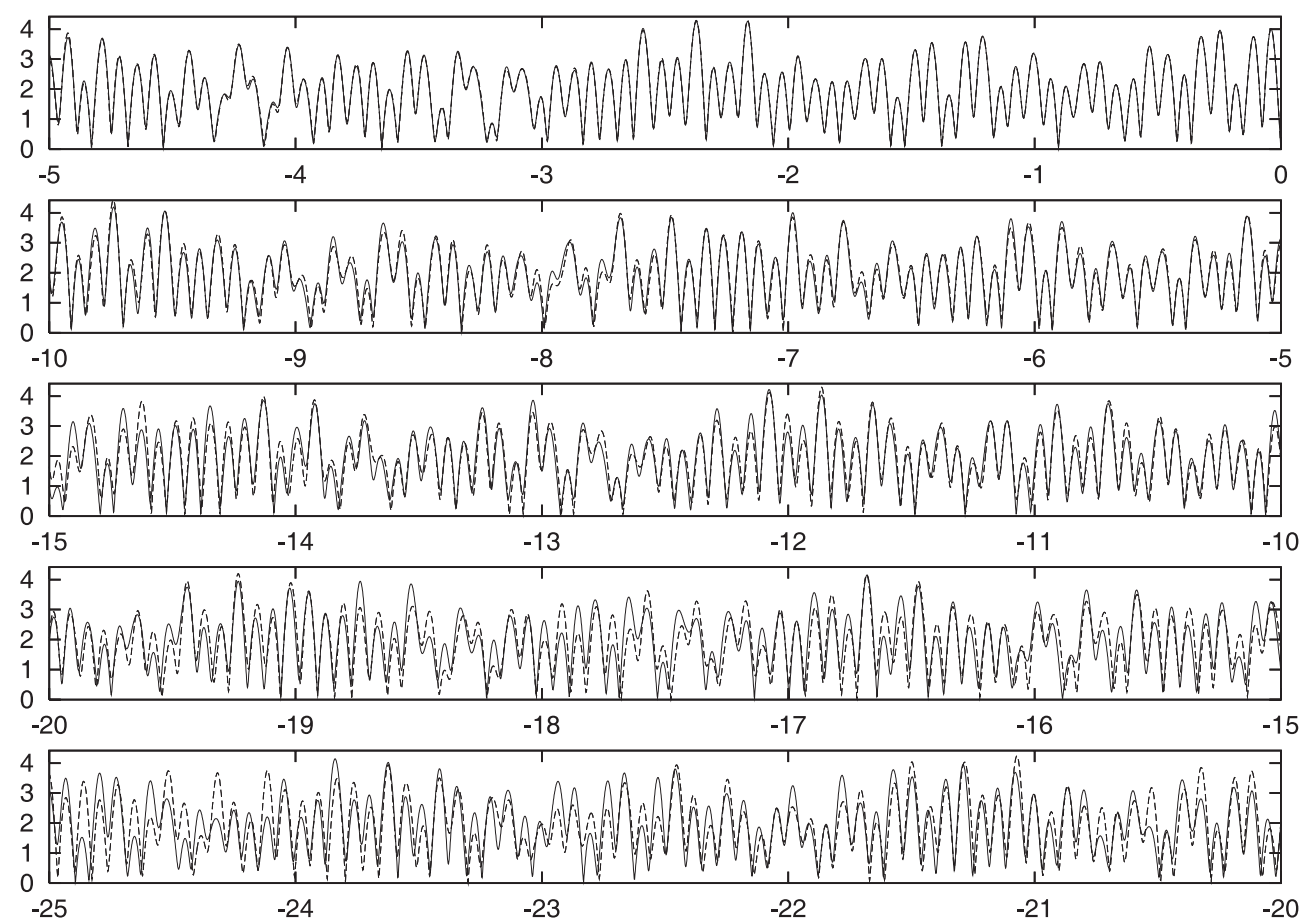

Fig. 6. Inclination (in degrees) of the Earth with respect to the fixed ecliptic J2000 over $25 \mathrm{Myr}$ in negative time from J2000. The solid line stands for the present solution La2004, while the dotted line is the eccentricity in the La93 solution (Laskar et al. 1993). The differences of the two solutions become noticeable after $15 \mathrm{Myr}$, and significantly different after $20 \mathrm{Myr}$.

has only very small variations (Fig. 11). Indeed, as shown by Laplace (1773) and Lagrange (1776), there are no secular variations of the semi-major axis of the planets at first order with respect to the masses, while some terms of higher order can be present (Haretu 1885). These terms are very small for the inner planets, but more visible in the solutions of the outer planets where the proximity of some mean motion resonances increases their contribution at second order with respect to the masses (Milani et al. 1987; Bretagnon \& Simon 1990). For the solution of the Earth, they are so small that they will not induce 
Table 3. Main secular frequencies $g_{i}$ and $s_{i}$ of La2004 determined over $20 \mathrm{Ma}$ for the four inner planets, and over $50 \mathrm{Ma}$ for the 5 outer planets (in $\operatorname{arcsec} \mathrm{yr}^{-1}$ ). $\Delta_{100}$ and $\Delta_{250}$ are the observed variations of the frequencies over respectively 100 and $250 \mathrm{Myr}$. In the last column, the period of the secular term are given.

\begin{tabular}{rcllr}
\hline \hline & $\left({ }^{\prime \prime} / \mathrm{yr}\right)$ & $\Delta_{100}$ & $\Delta_{250}$ & Period (yr) \\
\hline$g_{1}$ & 5.59 & 0.13 & 0.20 & 231843 \\
$g_{2}$ & 7.452 & 0.019 & 0.023 & 173913 \\
$g_{3}$ & 17.368 & 0.20 & 0.20 & 74620 \\
$g_{4}$ & 17.916 & 0.20 & 0.20 & 72338 \\
$g_{5}$ & 4.257452 & 0.000030 & 0.000030 & 304407 \\
$g_{6}$ & 28.2450 & 0.0010 & 0.0010 & 45884 \\
$g_{7}$ & 3.087951 & 0.000034 & 0.000048 & 419696 \\
$g_{8}$ & 0.673021 & 0.000015 & 0.000021 & 1925646 \\
$g_{9}$ & -0.34994 & 0.00063 & 0.0012 & 3703492 \\
\hline$s_{1}$ & -5.59 & 0.15 & 0.16 & 231843 \\
$s_{2}$ & -7.05 & 0.19 & 0.25 & 183830 \\
$s_{3}$ & -18.850 & 0.066 & 0.11 & 68753 \\
$s_{4}$ & -17.755 & 0.064 & 0.14 & 72994 \\
$s_{5}$ & 0.00000013 & 0.0000001 & 0.0000001 & \\
$s_{6}$ & -26.347855 & 0.000076 & 0.000087 & 49188 \\
$s_{7}$ & -2.9925259 & 0.000025 & 0.000025 & 433079 \\
$s_{8}$ & -0.691736 & 0.000010 & 0.000011 & 1873547 \\
$s_{9}$ & -0.34998 & 0.00051 & 0.0011 & 3703069 \\
\hline & & & &
\end{tabular}
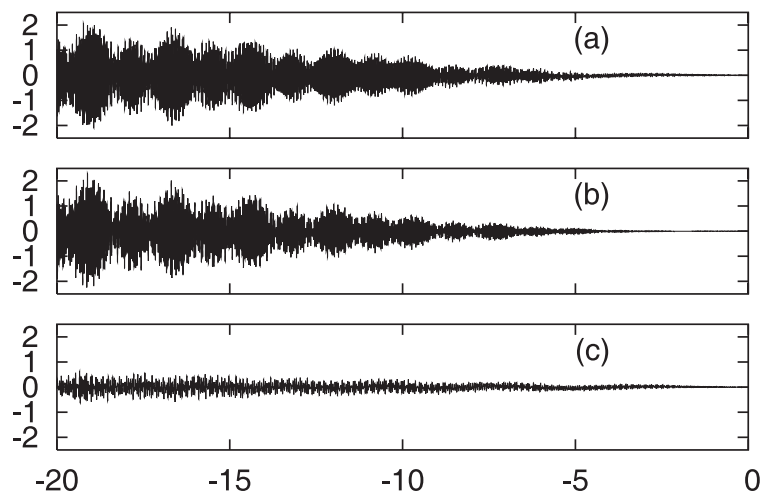

Fig. 7. Differences in obliquity (in degrees) La2004-La93(1, 1) versus time (in Myr) over $20 \mathrm{Myr}$ from present a). In La2004 both orbital and precession models have been changed. In b) only the precession model has been changed, while in c) only the orbital motion has been changed. It is thus clear that the main change in La2004 arise from the change of precession model.

any noticeable change of the mean Earth-Sun distance, or of the duration of the year in the geological past, at least over the past 250 millions of years.

It was first shown by Lagrange $(1774,1777)$ that the inclination and nodes of the planets suffer long term quasiperiodic variations. Shortly after, Laplace (1776) demonstrated that this was the same for the eccentricity and longitudes. Both computations were made using the linearization of the first order
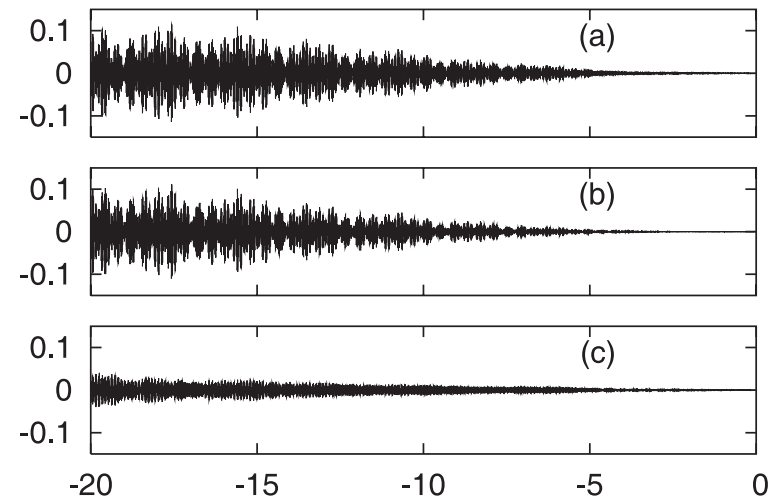

Fig. 8. Differences in climatic precession ( $e \sin \omega$, where $\omega=\varpi+\psi$ is the perihelion from the moving equinox) La2004-La93(1, 1) versus time (in Myr) over $20 \mathrm{Myr}$ from present a). In La2004 both orbital and precession models have been changed. In b) only the precession model has been changed, while in c) only the orbital motion has been changed. It is thus clear that the main change in La2004 arise from the change of precession model.

averaged equations of motion. In this approximation, if we use the complex notations

$z_{k}=e_{k} \exp \left(i \varpi_{k}\right) ; \quad \zeta_{k}=\sin \left(i_{k} / 2\right) \exp \left(i \Omega_{k}\right)$,

the equations of motion are given by a linear equation

$\frac{\mathrm{d}[x]}{\mathrm{d} t}=i A[x]$

where $[x]$ is the column vector $\left(z_{1}, \ldots, z_{9}, \zeta_{1}, \ldots, \zeta_{9}\right)$, and

$A=\left(\begin{array}{cc}A_{1} & 0 \\ 0 & A_{2}\end{array}\right)$

where the $9 \times 9$ matrices (for 9 planets) $A_{1}, A_{2}$ depend on the planetary masses and semi-major axis of the planets. The resolution of these equations is now classical, and is obtained through the diagonalization of the matrix $A$ by the change of coordinates into proper modes $u$

$[x]=S[u]$.

The solution in the proper modes $[u]=\left(z_{1}^{\bullet}, \ldots, z_{9}^{\bullet}, \zeta_{1}^{\bullet}, \ldots, \zeta_{9}^{\bullet}\right)$ is then given by the diagonal system

$\frac{\mathrm{d}[u]}{\mathrm{d} t}=i D[u]$

where $D=S^{-1} A S$ is a diagonal matrix $D=$ $\operatorname{Diag}\left(g_{1}, g_{2}, \ldots, g_{9}, s_{1}, s_{2}, \ldots, s_{9}\right)$, where all the eigenvalues $g_{k}, s_{k}$ are real. The solutions of the proper modes $z_{k}^{\bullet}, \zeta_{k}^{\bullet}$ are

$z_{k}^{\bullet}(t)=z_{k}^{\bullet}(0) \exp \left(i g_{k} t\right) ; \quad \zeta_{k}^{\bullet}(t)=\zeta_{k}^{\bullet}(0) \exp \left(i s_{k} t\right)$.

The solutions in the elliptical variables $z_{k}, \zeta_{k}$ are then given as linear combinations of the proper modes (19), and becomes quasiperiodic functions of the time $t$, that is sums of pure periodic terms with independent frequencies.

$z_{k}=\sum_{k=1}^{9} \alpha_{k j} \mathrm{e}^{i g_{k} t} ; \quad \zeta_{k}=\sum_{k=1}^{9} \beta_{k j} \mathrm{e}^{i s_{k} t}$ 
Lagrange $(1781,1782)$ actually did this computation for a planetary system including Mercury, Venus, Earth, Mars, Jupiter and Saturn. Pontécoulant (1834) extended it to the 7 known planets of the time, but with several errors in the numerical constants. The first precise solution for the Earth was given by Le Verrier (1856) for 7 planets, and was later on extended by Stockwell (1873) with the addition of Neptune. The validity of the linear approximation (17) was also questioned and subsequent improvements introduced some additional contribution of higher degree in eccentricity and inclination in Eq. (17), as well as terms of higher order with respect to the planetary masses in the averaged equations (Hill 1897; Brouwer \& Van Woerkom 1950; Bretagnon 1974; Duriez 1977; Laskar $1985)$. With the addition of additional non linear terms $B(x, \bar{x})$ (of degree $\geq 3$ in eccentricity and inclination), the secular equations

$$
\frac{\mathrm{d}[x]}{\mathrm{d} t}=i A[x]+B(x, \bar{x})
$$

are no longer integrable. Nevertheless, one can formally perform a Birkhoff normalization of Eq. (23) and after truncation, obtain some quasiperiodic expressions

$$
\begin{aligned}
& z_{j}^{*}(t)=\sum_{k=1}^{9} a_{j k} \mathrm{e}^{i g_{k} t}+\sum_{(k)} a_{j(k)} \mathrm{e}^{i<(k), v>t} \\
& \zeta_{j}^{*}(t)=\sum_{k=1}^{9} b_{j k} \mathrm{e}^{i S_{k} t}+\sum_{(k)} b_{j(k)} \mathrm{e}^{i<(k), v>t}
\end{aligned}
$$

where $(k)=\left(k_{1}, \ldots, k_{18}\right) \in \mathbb{Z}^{18}, v=\left(g_{1}, \ldots, g_{9}, s_{1}, \ldots, s_{9}\right)$, and $\langle(k), v\rangle=k_{1} g_{1}+\ldots+k_{9} g_{9}+k_{10} s_{1}+\ldots+k_{18} s_{9}$. The quasiperiodic expressions $z_{j}^{*}(t), \zeta_{j}^{*}(t)$, have been computed at various orders and degrees in eccentricity and inclination of expansion (Hill 1897; Bretagnon 1974; Duriez 1977; Laskar 1984, 1985), but as forecasted by Poincaré (1893), it was found that when all the main planets are taken into account, these formal expansions do not converge (Laskar 1984), for initial conditions close to the initial conditions of the Solar System, even at the lowest orders, and thus will not provide good approximation of the Solar System motion over very long time. In other terms, it appears that the actual solution of the Solar System is not close to a KAM tori (see Arnold et al. 1988) of quasiperiodic solutions.

Over a finite time of a few millions of years, one can still numerically integrate the equation of motion, and then search through frequency analysis (Laskar 1990, 1999, 2003) for a quasiperiodic approximation of the solution of the form (23). The non regular behavior of the solution will induce a drift of the frequencies in time, related to the chaotic diffusion of the trajectories (Laskar 1990, 1993, 1999).

In Table 3, the fundamental frequencies of the solution La2004 have been computed by frequency analysis over $20 \mathrm{Myr}$ of the proper modes $\left(z_{1}^{\bullet}, \ldots, z_{4}^{\bullet}, \zeta_{1}^{\bullet}, \ldots, \zeta_{4}^{\circ}\right)$ related to the inner planets (Mercury, Venus, Earth, Mars), and over $50 \mathrm{Myr}$ for the proper modes $\left(z_{5}^{\bullet}, \ldots, z_{9}^{\circ}, \zeta_{5}^{\bullet}, \ldots, \zeta_{9}^{\bullet}\right)$ associated to the outer planets (Jupiter, Saturn, Uranus, Neptune, Pluto) (Table 3). This difference reflects the fact that the main source of chaotic behavior comes from overlap of secular resonances in the inner planet system, and that the outer planets secular systems is more regular than the inner one. Actually, in Cols. 2 and 3 of Table 3, we give the maximum observed in the variation of the secular frequencies over 100 and $250 \mathrm{Myr}$, in both positive and negative time, for our nominal solution La2004. These figures are in very good agreement with the equivalent quantities given in Table X of (Laskar 1990) for the integration of the secular equations. The number of digits of the fundamental frequencies in Table 3 is different for each frequency and reflects the stability of the considered frequency with time. Additionally, we have given in Figs. 9 and 10 the actual evolution of the secular frequencies obtained with a sliding window with a step size of $1 \mathrm{Myr}$ (compare to Laskar 1990, Figs. 8 and 9).

\section{Analytical approximations}

\subsection{Orbital motion}

It is interesting for practical use to have an analytical expression for the main orbital quantities of the Earth. From the numerical values of La2004, we have performed a frequency analysis (Laskar 1990, 1999, 2003) in order to obtain a quasiperiodic approximation of the solutions over a few Myr. In order to be consistent with the remaining part of the paper, we chose a time interval covering $20 \mathrm{Myr}$. As we are mostly interested over negative time, we made the analysis from $-15 \mathrm{Myr}$ to $+5 \mathrm{Myr}$, as usually, the precision of the approximation decreases at the edges of the time interval. The analysis of the eccentricity variables $z=e \exp i \varpi$ is given in Table $4 . z$ is obtained as

$z=\sum_{k=1}^{26} b_{k} \mathrm{e}^{i\left(\mu_{k} t+\varphi_{k}\right)}$

It should be noted that in the present complex form, the approximation is much more precise than what would give an equivalent quasiperiodic approximation of the eccentricity. Even with twice the number of periodic terms, the direct approximation of the eccentricity is not as good as the approximation obtained in complex variables. Moreover, here we obtain at the same time the approximation of the longitude of perihelion of the Earth from the fixed J2000 equinox ( $\varpi)$. The comparison of this approximation, limited to only 26 terms, to the actual solution for the eccentricity of the Earth, is plotted in Fig. 12 (top).

Nevertheless, for information, we will give also here the leading terms in the expansion of the eccentricity as they may be useful in paleoclimate studies (Table 6). The three leading terms in eccentricity are well known in paleoclimate studies $g_{2}-g_{5}$ (405 kyr period), $g_{4}-g_{5}$ (95 kyr period), and $g_{4}-g_{2}$ (124 kyr period).

The solution for the inclination variables $\zeta=\sin i / 2 \exp i \Omega$ where $i, \Omega$ are the Earth inclination and longitude of node with respect to the fixed ecliptic and equinox J2000, limited to 24 quasiperiodic terms

$\zeta=\sum_{k=1}^{24} a_{k} \mathrm{e}^{i\left(v_{k} t+\phi_{k}\right)}$ 


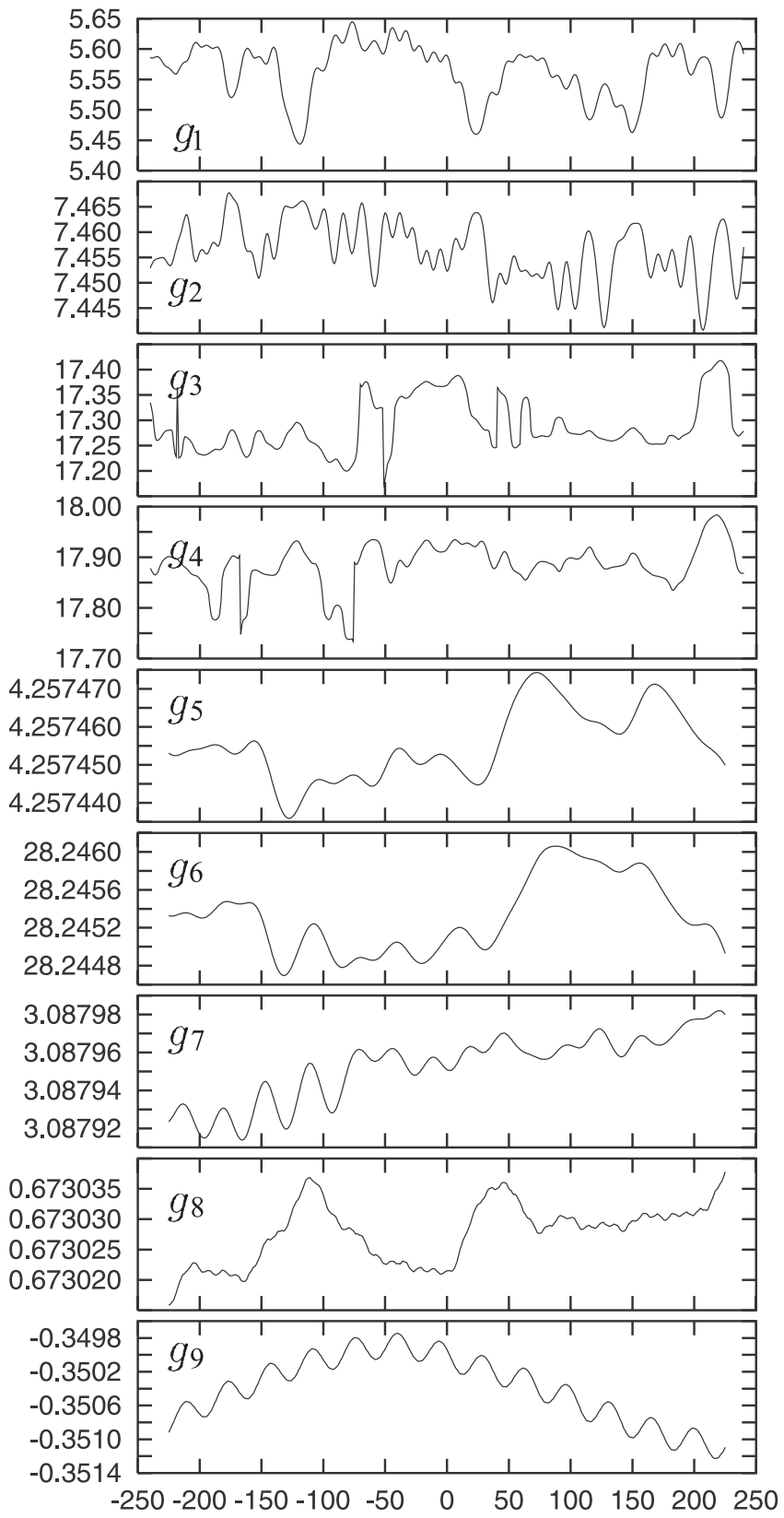

Fig. 9. Variation of the secular frequencies $g_{1-9}$ from -250 to $+250 \mathrm{Myr}$. The frequencies are computed over $20 \mathrm{Myr}$ for $g_{1-4}$ and over $50 \mathrm{Myr}$ for $g_{5-9}$, after transformation of elliptical elements to proper modes (Laskar 1990).

is given in Table 5. The comparison of this quasiperiodic approximation with the complete solution is given in Fig. 12 (bottom). It should be noted that in Table 5, the first 22 terms are the terms of largest amplitude in the frequency decomposition of the inclination variable $\zeta$, but the two last terms, of frequency $v_{23}$ and $v_{24}$ are of much smaller amplitude. They have been kept in the solution, as they are close to the resonance with the main precession frequency.
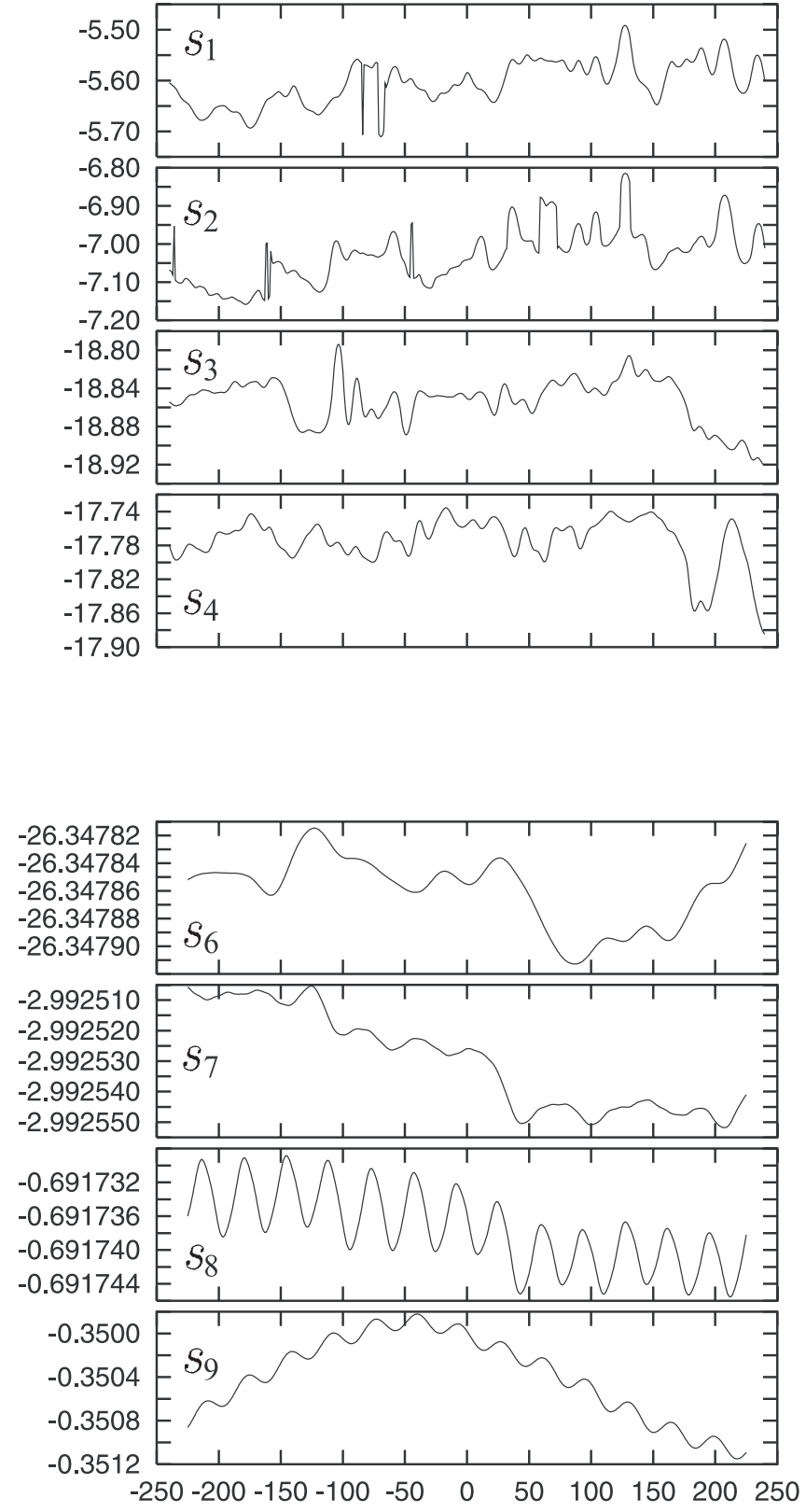

Fig. 10. Variation of the secular frequencies $s_{1-9}$ from -250 to $+250 \mathrm{Myr}$. The frequencies are computed over $20 \mathrm{Myr}$ for $s_{1-4}$ and over $50 \mathrm{Myr}$ for $s_{6-9}$, after transformation of elliptical elements to proper modes (Laskar 1990).

\subsection{Obliquity and precession}

The solutions for precession and obliquity are obtained through the precession Eq. (6). In absence of planetary perturbations, the obliquity is constant $\left(\varepsilon=\varepsilon_{0}\right)$, and

$\frac{\mathrm{d} \psi}{\mathrm{d} t}=\alpha \cos \varepsilon_{0}$

We have thus $\psi=\psi_{0}+p t$ where $p=\alpha \cos \varepsilon_{0}$ is the precession frequency. This can be considered as a solution of order zero. 
Table 4. Frequency decomposition of $z=e \exp i \varpi$ for the Earth on the time interval $[-15,+5]$ Myr (Eq. (25)).

\begin{tabular}{rlrrr}
\hline \hline$n$ & & $\mu_{k}\left({ }^{\prime \prime} / \mathrm{yr}\right)$ & $b_{k}$ & $\varphi_{k}($ degree $)$ \\
\hline 1 & $g_{5}$ & 4.257564 & 0.018986 & 30.739 \\
2 & $g_{2}$ & 7.456665 & 0.016354 & -157.801 \\
3 & $g_{4}$ & 17.910194 & 0.013055 & 140.577 \\
4 & $g_{3}$ & 17.366595 & 0.008849 & -55.885 \\
5 & $g_{1}$ & 5.579378 & 0.004248 & 77.107 \\
6 & & 17.112064 & 0.002742 & 47.965 \\
7 & & 17.654560 & 0.002386 & 61.025 \\
8 & & 6.954694 & 0.001796 & -159.137 \\
9 & $2 g_{3}-g_{4}$ & 16.822731 & 0.001908 & 105.698 \\
10 & $g_{6}$ & 28.244959 & 0.001496 & 127.609 \\
11 & & 18.452681 & 0.001325 & 156.113 \\
12 & & 5.446730 & 0.001266 & -124.155 \\
13 & & 18.203535 & 0.001165 & -92.112 \\
14 & & 7.325741 & 0.001304 & -175.144 \\
15 & & 7.060100 & 0.001071 & 2.423 \\
16 & & 7.587926 & 0.000970 & 34.169 \\
17 & & 5.723726 & 0.001002 & 112.564 \\
18 & & 16.564805 & 0.000936 & 24.637 \\
19 & & 16.278532 & 0.000781 & -90.999 \\
20 & & 18.999613 & 0.000687 & 0.777 \\
21 & & 3.087424 & 0.000575 & 120.376 \\
22 & & 17.223297 & 0.000577 & -113.456 \\
23 & & 6.778196 & 0.000651 & 117.184 \\
24 & & 6.032715 & 0.000416 & 174.987 \\
25 & & 6.878659 & 0.000497 & 95.424 \\
26 & & 5.315656 & 0.000392 & 33.255 \\
\hline & & & & \\
1 & & &
\end{tabular}

If we keep only the terms of degree one in inclination in Eqs. (6), we obtain the solution of order one

$\frac{\mathrm{d} \varepsilon}{\mathrm{d} t}=2(\dot{p} \sin \psi-\dot{q} \cos \psi)=2 \mathcal{R} e\left(\dot{\zeta} \mathrm{e}^{i \psi}\right)$.

With the quasiperiodic approximation

$\zeta=\sum_{k=1}^{N} a_{k} \mathrm{e}^{i\left(v_{k} t+\phi_{k}\right)}$

of Table 5 , the first order solution in obliquity will be a similar quasiperiodic function

$\varepsilon=\varepsilon_{0}+2 \sum_{k=1}^{N} \frac{a_{k} v_{k}}{v_{k}+p} \cos \left(\left(v_{k}+p\right) t+\phi_{k}+\psi_{0}\right)$,

with a similar form for the solution of precession $\psi$. This first order approximation will only be valid when we are far from resonance, that is $\left|v_{k}+p\right| \gg 0$. This is not the case for the last

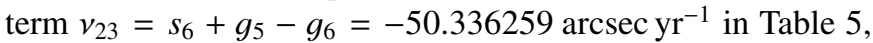
as $p \approx 50.484 \operatorname{arcsec}^{-1} \mathrm{r}^{-1}$. This resonant term can lead to a

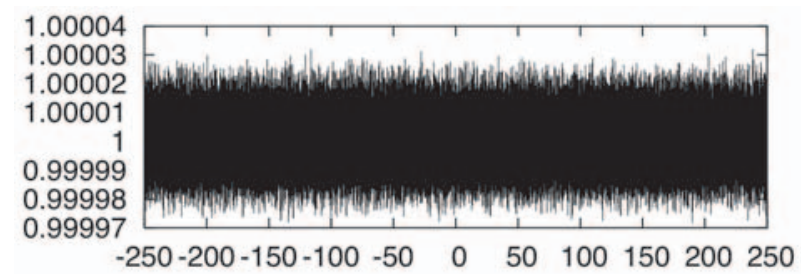

Fig. 11. Variation of the semi-major axis of the Earth-Moon barycenter (in AU) from -250 to $+250 \mathrm{Myr}$.

Table 5. Frequency decomposition of $\zeta=\sin i / 2 \exp i \Omega$ for the Earth on the time interval $[-15,+5]$ Myr (Eq. (26)).

\begin{tabular}{rlrrr}
\hline \hline$n$ & & $v_{k}\left({ }^{\prime} / \mathrm{yr}\right)$ & $a_{k}$ & $\phi_{k}$ (degree) \\
\hline 1 & $s_{5}$ & -0.000001 & 0.01377449 & 107.581 \\
2 & $s_{3}$ & -18.845166 & 0.00870353 & -111.310 \\
3 & $s_{1}$ & -5.605919 & 0.00479813 & 4.427 \\
4 & $s_{2}$ & -7.050665 & 0.00350477 & 130.826 \\
5 & $s_{4}$ & -17.758310 & 0.00401601 & -77.666 \\
6 & & -18.300842 & 0.00262820 & -93.287 \\
7 & & -7.178497 & 0.00189297 & -65.292 \\
8 & & -6.940312 & 0.00164641 & -66.106 \\
9 & & -6.817771 & 0.00155131 & -46.436 \\
10 & & -19.389947 & 0.00144918 & 50.158 \\
11 & & -5.481324 & 0.00154505 & 23.486 \\
12 & & -26.347880 & 0.00133215 & 127.306 \\
13 & & -19.111118 & 0.00097602 & 159.658 \\
14 & & -2.992612 & 0.00088810 & 140.098 \\
15 & & -6.679492 & 0.00079348 & -43.800 \\
16 & & -5.835373 & 0.00073058 & -160.927 \\
17 & & -0.691879 & 0.00064133 & 23.649 \\
18 & & -6.125755 & 0.00049598 & 143.759 \\
19 & & -5.684257 & 0.00054320 & 138.236 \\
20 & & -18.976417 & 0.00040739 & -105.209 \\
21 & & -7.290771 & 0.00041850 & 106.192 \\
22 & & -5.189414 & 0.00033868 & 50.827 \\
23 & $s_{6}+g_{5}-g_{6}$ & -50.336259 & 0.00000206 & -150.693 \\
24 & & -47.144010 & 0.00000023 & -167.522 \\
\hline & & & & \\
\hline & & & & \\
& & & &
\end{tabular}

significative change in the obliquity variations, and have been studied in detail in Laskar et al. (1993).

Another difficulty, arising with the obliquity solution, is due to the dissipation in the Earth-Moon system, which induces a significant variation of the precession frequency with time, as the Earth rotation $\omega$ slows down, and the Earth-Moon distance $a_{\mathrm{M}}$ increases.

We have plotted in Fig. 14 the evolution of the obliquity of the Earth from -250 to +250 Myr. The effect of the tidal dissipation is clearly visible on this timescale, and there is a general increase of the obliquity from $-250 \mathrm{Myr}$ to present time. In positive time, there is an obvious singularity in the obliquity, with a decrease of about 0.4 degree. This results from the 

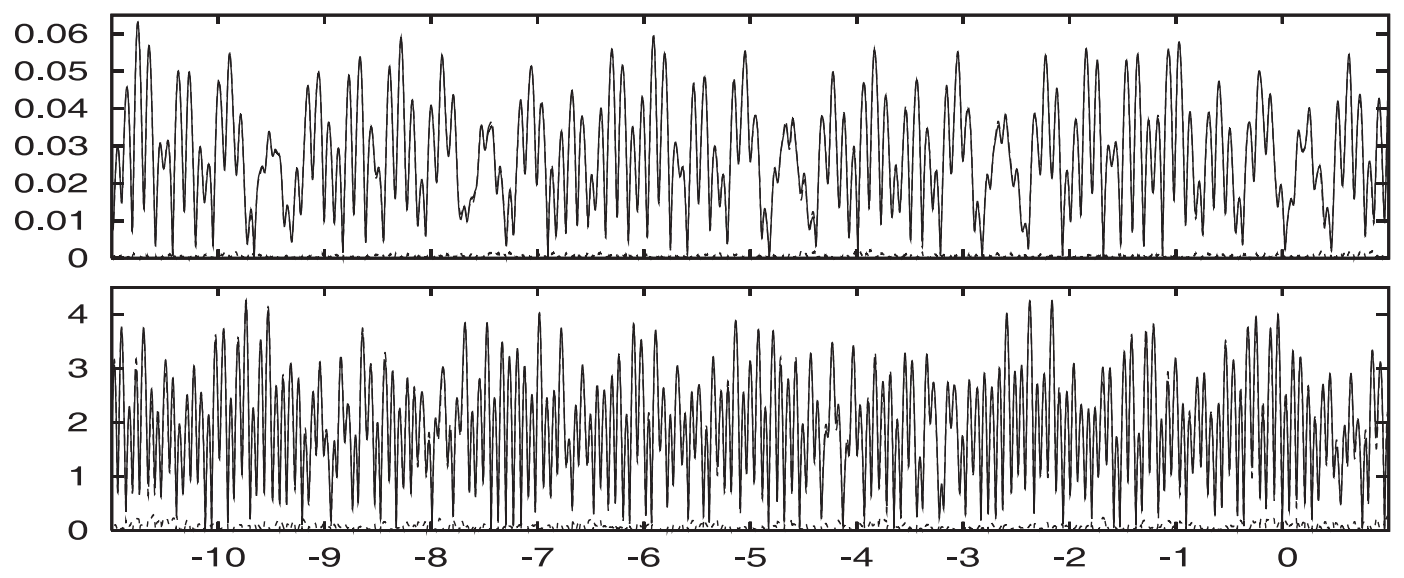

Fig. 12. (Top) Eccentricity of the Earth from -11 to +1 Myr. The solid curve is the solution La2004. Almost completely hidden behind the solid curve is a dashed curve representing the quasiperiodic approximation of Table 4 with 26 periodic terms. (Bottom) Inclination of the Earth from -11 to +1 Myr. The solid curve is the solution La2004. The dashed line (almost identical to the solid curve) is the quasiperiodic approximation of Table 5 with 24 periodic terms. On both plots, as the two curves are nearly identical, the difference of the two solutions is also plotted in dotted curve.

crossing of the resonance with the term $s_{6}+g_{5}-g_{6}$ that was already described in a different context in Laskar et al. (1993). After this sudden decrease, the obliquity increases again, with roughly the same trend. An additional (but smaller singularity is observable around $+150 \mathrm{Myr}$, that corresponds to the crossing of the resonance with the term $v_{24}$ (Table 5). The averaged obliquity can be well approximated in negative time with the expression

$\varepsilon_{\mathrm{M}}=23.270773+2.011295 T$

where $\varepsilon_{\mathrm{M}}$ is in degree, and $T$ in billion of years (Gyr). In the interval [+20 Myr, +130 Myr] this approximation becomes

$\varepsilon_{\mathrm{M}}=22.893171+1.952715 T$

so the main effect of the crossing of the resonance is a decrease of 0.377602 degree of the obliquity. This effect can be easily understood with the analytical integration of a simple model, in terms of Fresnel integrals (see Appendix A).

\subsection{Approximation for the obliquity}

As for the solutions of the orbital elements, we have obtained an approximation formula for the evolution of the obliquity of the Earth over the period from $-15 \mathrm{Myr}$ to $+5 \mathrm{Myr}$. Obtaining this solution presents some additional difficulties because of the dissipative effects in the Earth-Moon system that induces a significative change of the precession frequency. The obliquity is obtained on the form

$\varepsilon=\varepsilon_{0}+\sum_{k=1}^{N} a_{k}^{\prime} \cos \left(\left(v_{k}^{\prime}+p_{1} t\right) t+\phi_{k}^{\prime}\right)$,

where $\varepsilon_{0}=23.254500$ degrees, and the coefficients $a_{k}^{\prime}, v_{k}^{\prime}, \phi_{k}^{\prime}$ are given in Table 7. The coefficent $p_{1}$ (Eq. (35)) results from the secular change of the precession frequency (34) due to tidal dissipation (see next).

The comparison with the full solution is given in Fig. 15, over selected periods of time, from -11 Myr to the present.
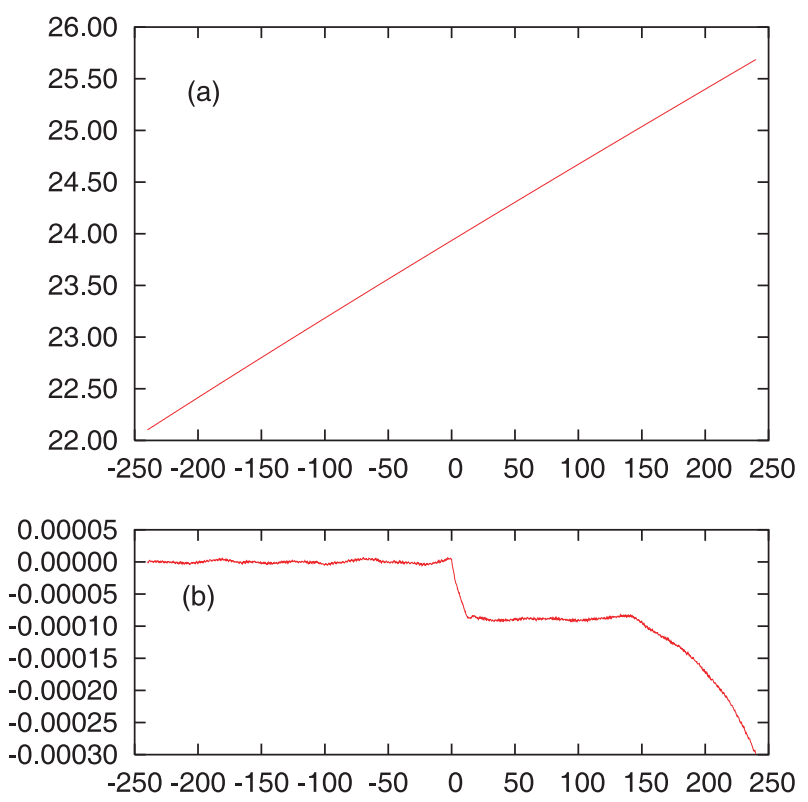

Fig. 13. a) Evolution of length of the day for the Earth, in hours, from -250 to +250 Myr. b) Residuals with the fit of the averaged solution with the polynomial expression (41).

In Table 7, the terms of frequency $v_{k}^{\prime}$ are recognized as combinations of the form $p+v_{k}$, where $p$ is the precession frequency, and $v_{k}$ a secular frequency from the secular motion of the orbital plane (from Table 5). The approximation is made over the interval $[-15 \mathrm{Myr},+2 \mathrm{Myr}]$ with $p=p_{0}+p_{1} T$, where $p_{0}$ and $p_{1}$ are taken from Eq. (35).

\subsection{Approximation for the precession}

The full approximate solution for the precession is more complicated because of the presence of the secular resonance $s_{6}+$ $g_{5}-g_{6}$. In fact, the proximity of this resonance is responsible for the largest periodic term in the expression of the precession 
Table 6. Frequency decomposition of the eccentricity of the Earth on the time interval $[-15,+5]$ Myr. For all computations, the data of Table 4 should be preferred. Here, $e=e_{0}+\sum_{k=1}^{20} b_{k}^{\prime} \cos \left(\mu_{k}^{\prime} t+\varphi_{k}\right)$ with $e_{0}=0.0275579$. For each term, in the first column is the corresponding combination of frequencies where $g_{i}$ are the fundamental frequencies (Table 3), and $\mu_{j}$ the frequencies of the terms in $z$ from Table 4.

\begin{tabular}{rcrrrr}
\hline \hline$k$ & & $\mu_{k}^{\prime}\left({ }^{\prime \prime} / \mathrm{yr}\right)$ & $P(\mathrm{yr})$ & $b_{k}^{\prime}$ & $\varphi_{k}^{\prime}($ degree $)$ \\
\hline 1 & $g_{2}-g_{5}$ & 3.199279 & 405091 & 0.010739 & 170.739 \\
2 & $g_{4}-g_{5}$ & 13.651920 & 94932 & 0.008147 & 109.891 \\
3 & $g_{4}-g_{2}$ & 10.456224 & 123945 & 0.006222 & -60.044 \\
4 & $g_{3}-g_{5}$ & 13.109803 & 98857 & 0.005287 & -86.140 \\
5 & $g_{3}-g_{2}$ & 9.909679 & 130781 & 0.004492 & 100.224 \\
6 & $\mu_{7}-\mu_{6}$ & 0.546076 & 2373298 & 0.002967 & -168.784 \\
7 & $g_{1}-g_{5}$ & 1.325696 & 977600 & 0.002818 & 57.718 \\
8 & $g_{4}-g_{1}$ & 12.325286 & 105150 & 0.002050 & 49.546 \\
9 & $g_{2}-g_{5}+\mu_{6}-\mu_{7}$ & 2.665308 & 486248 & 0.001971 & 148.774 \\
10 & $g_{2}-g_{1}$ & 1.883616 & 688038 & 0.001797 & 137.155 \\
11 & $\mu_{6}-g_{5}$ & 12.856520 & 100805 & 0.002074 & 24.487 \\
12 & $g_{2}+g_{4}-2 g_{5}$ & 16.851127 & 76909 & 0.001525 & 102.380 \\
13 & $\mu_{6}-g_{2}$ & 9.650041 & 134300 & 0.001491 & -167.676 \\
14 & $2 g_{3}-g_{4}-g_{5}$ & 12.563233 & 103158 & 0.001316 & 69.234 \\
15 & $2 g_{4}-g_{2}-g_{3}$ & 10.975914 & 118077 & 0.001309 & -51.163 \\
16 & $g_{1}-g_{3}$ & 11.788709 & 109936 & 0.001300 & -146.081 \\
17 & $\mu_{7}-g_{2}$ & 10.194846 & 127123 & 0.001306 & -139.827 \\
18 & $g_{3}+g_{4}-g_{2}-g_{5}$ & 23.562689 & 55002 & 0.001261 & 30.098 \\
19 & $\mu_{7}-g_{5}$ & 13.398519 & 96727 & 0.001344 & 27.612 \\
20 & $g_{2}+g_{4}-g_{3}-g_{5}$ & 3.742221 & 346318 & 0.001058 & 178.662 \\
\hline
\end{tabular}

angle (Fig. 16). The precession angle can thus be approximate by ( $\psi$ in arcsec, $t$ in years)

$$
\begin{aligned}
\psi= & +49086+p_{0} t+p_{1} t^{2} \\
& +42246 \frac{v_{0}^{2}}{\left(v_{0}+2 p_{1} t\right)^{2}} \cos \left(v_{0} t+p_{1} t^{2}+\phi_{0}\right)
\end{aligned}
$$

where

$$
\begin{aligned}
& v_{0}=0.150019^{\prime \prime} / \mathrm{yr} \\
& p_{0}=50.467718^{\prime \prime} / \mathrm{yr} \\
& p_{1}=-13.526564^{-9 \prime} / \mathrm{yr}^{2} \\
& \phi_{0}=171.424 \text { degree. }
\end{aligned}
$$

It should be noted that in this formula, the cosine term is an approximation for a more complicated expression that arises from a double integration of the term $\cos \left(v_{0} t+p_{1} t^{2}+\phi_{0}\right)$ in the expression of the derivative of the obliquity (6). In order to obtain an expression as simple as possible, without expressions involving Fresnel integrals (Appendix A), we had to restrain its interval of validity to $[-15,+2]$ Myr.

For paleoclimate studies, the usual quantity that relates more directly to insolation is the climatic precession $e \sin \bar{\omega}$, where $\bar{\omega}=\psi+\varpi$ is the longitude of the perihelion from the moving equinox. We have thus

$e \sin \bar{\omega}=\mathfrak{J}\left(z \mathrm{e}^{i \psi(t)}\right)$,

where the decomposition of $z=e \exp (i \varpi)$ is provided in Table 4 , and the precession angle $\psi$ is given by Eq. (34).

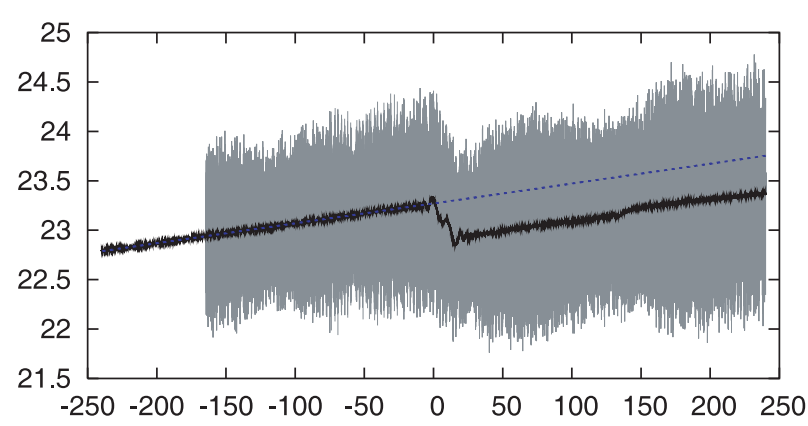

Fig. 14. Evolution of the obliquity of the Earth in degrees, from -250 to +250 Myr. The grey zone is the actual obliquity, while the black curve is the averaged value of the obliquity over $0.5 \mathrm{Myr}$ time intervals. The dotted line is a straight line fitted to the average obliquity in the past.

The comparison of this approximation with the complete solution from -11 to $+1 \mathrm{Myr}$ is given in Fig. 17. The solution for climatic precession is thus on the form

$e \sin \bar{\omega}=\sum_{k=1}^{20} b_{k} \sin \left(\mu_{k} t+\varphi_{k}+\psi(t)\right)$

where $b_{k}, \mu_{k}, \varphi_{k}$ are from Table 4 , and where $\psi(t)$ is given by Eq. (34). The frequencies of the terms of the climatic precession are thus of the form

$\mu_{k}^{\prime \prime}=\mu_{k}+p+p_{1} t$,

where $p, p_{1}$ are given in Eqs. (34) and (35). 
Table 7. Approximation for the obliquity of the Earth, following Eq. (33). This expression is not strictly quasiperiodic, because of the presence of the dissipative term $p_{1}$ in the evolution of the precesion frequency (33).

\begin{tabular}{|c|c|c|c|c|c|}
\hline$k$ & & $v_{k}^{\prime}\left({ }^{\prime \prime} / \mathrm{yr}\right)$ & $P(\mathrm{yr})$ & $a_{k}^{\prime}$ & $\phi_{k}^{\prime}(\mathrm{d})$ \\
\hline 1 & $p+s_{3}$ & 31.626665 & 40978 & 0.582412 & 86.645 \\
\hline 2 & $p+s_{4}$ & 32.713667 & 39616 & 0.242559 & 120.859 \\
\hline 3 & $p+s_{6}$ & 24.124241 & 53722 & 0.163685 & -35.947 \\
\hline 4 & $p+v_{6}$ & 32.170778 & 40285 & 0.164787 & 104.689 \\
\hline 5 & $p+v_{10}$ & 31.081475 & 41697 & 0.095382 & -112.872 \\
\hline 6 & $p+v_{20}$ & 31.493347 & 41152 & 0.094379 & 60.778 \\
\hline 7 & $p+s_{6}+g_{5}-g_{6}$ & 0.135393 & 9572151 & 0.087136 & 39.928 \\
\hline 8 & $p+s_{2}$ & 43.428193 & 29842 & 0.064348 & -15.130 \\
\hline 9 & $p+s_{1}$ & 44.865444 & 28886 & 0.072451 & -155.175 \\
\hline 10 & & 31.756641 & 40810 & 0.080146 & -70.983 \\
\hline 11 & $p+v_{13}$ & 31.365950 & 41319 & 0.072919 & 10.533 \\
\hline 12 & & 32.839446 & 39465 & 0.033666 & -31.614 \\
\hline 13 & & 32.576100 & 39784 & 0.033722 & 77.554 \\
\hline 14 & & 32.035200 & 40455 & 0.030677 & 71.757 \\
\hline 15 & $p+v_{8}$ & 43.537092 & 29768 & 0.039351 & 145.835 \\
\hline 16 & $p+v_{7}$ & 43.307432 & 29926 & 0.030375 & 160.109 \\
\hline 17 & $p+v_{9}$ & 43.650496 & 29690 & 0.024733 & 144.926 \\
\hline 18 & & 31.903983 & 40622 & 0.025201 & -173.656 \\
\hline 19 & & 30.945195 & 41880 & 0.021615 & -144.933 \\
\hline 20 & & 23.986877 & 54030 & 0.021565 & -79.670 \\
\hline 21 & & 24.257837 & 53426 & 0.021270 & -178.441 \\
\hline 22 & & 32.312463 & 40108 & 0.021851 & -24.566 \\
\hline 23 & & 44.693687 & 28997 & 0.014725 & 124.744 \\
\hline
\end{tabular}

\subsection{Earth-Moon system}

In Fig. 18, the evolution of the Earth-Moon semi-major $a_{\mathrm{M}}$ axis is plotted over $250 \mathrm{Myr}$ in positive and negative time. The grey area corresponds to the short period variations of the EarthMoon distance, obtained in the full integration of the system, while the black curve is the integration of the averaged equations that is used for the precession computations. The agreement between the two solutions is very satisfying.

We have searched for a polynomial approximation for the secular evolution of $a_{\mathrm{M}}$, in order to provide a useful formula for simple analytical computations. In fact, the Earth-Moon evolution is driven by the tidal dissipation equations (Sect. 4.1), that involve the obliquity of the planet. The important singularity of the obliquity in the future (see previous section) prevents from using a single approximation over the whole time interval, and we have used a different polynomial in positive and negative time.

$$
\begin{aligned}
& a_{\mathrm{M}}^{-}=60.142611 \quad a_{\mathrm{M}}^{+}=60.142611 \\
& +6.100887 T \quad+6.120902 T \\
& -2.709407 T^{2} \quad-2.727887 T^{2} \\
& +1.366779 T^{3} \quad+1.614481 T^{3} \\
& -1.484062 T^{4} \quad-0.926406 T^{4}
\end{aligned}
$$

where $T$ is in billions of years (Gyr), and $a_{\mathrm{M}}^{+-}$in Earth radius (see Table 1). In the residuals plot (Fig. 18b), we can check that this polynomial approximation is very precise for negative time, but in positive time, we have an additional singularity around $150 \mathrm{Myr}$, resulting from the secular resonance of the precession frequency with $v_{24}$ (Table 5).

In the same way, it is possible to approximate the evolution of the precession frequency of the Earth $p$ as the Moon goes away and the rotation of the Earth slows down with time. The polynomial approximation of the precession frequency is then in $\operatorname{arcsec} \mathrm{yr}^{-1}$, with $T$ in Gyr

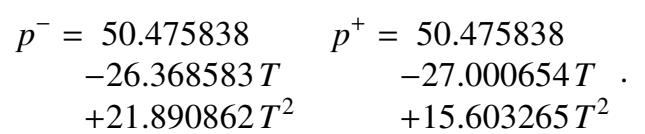

It should be said that the initial value is kept equal for both positive and negative time to avoid discontinuities. It is clear from Fig. 19 that for the period between +30 and +130 Myr, a better approximation could be obtained by adding to the constant part the offset $+0.135052 \operatorname{arcsec} \mathrm{yr}^{-1}$. It is interesting to note again the dramatic influence of the resonance $v_{23}$ on the 

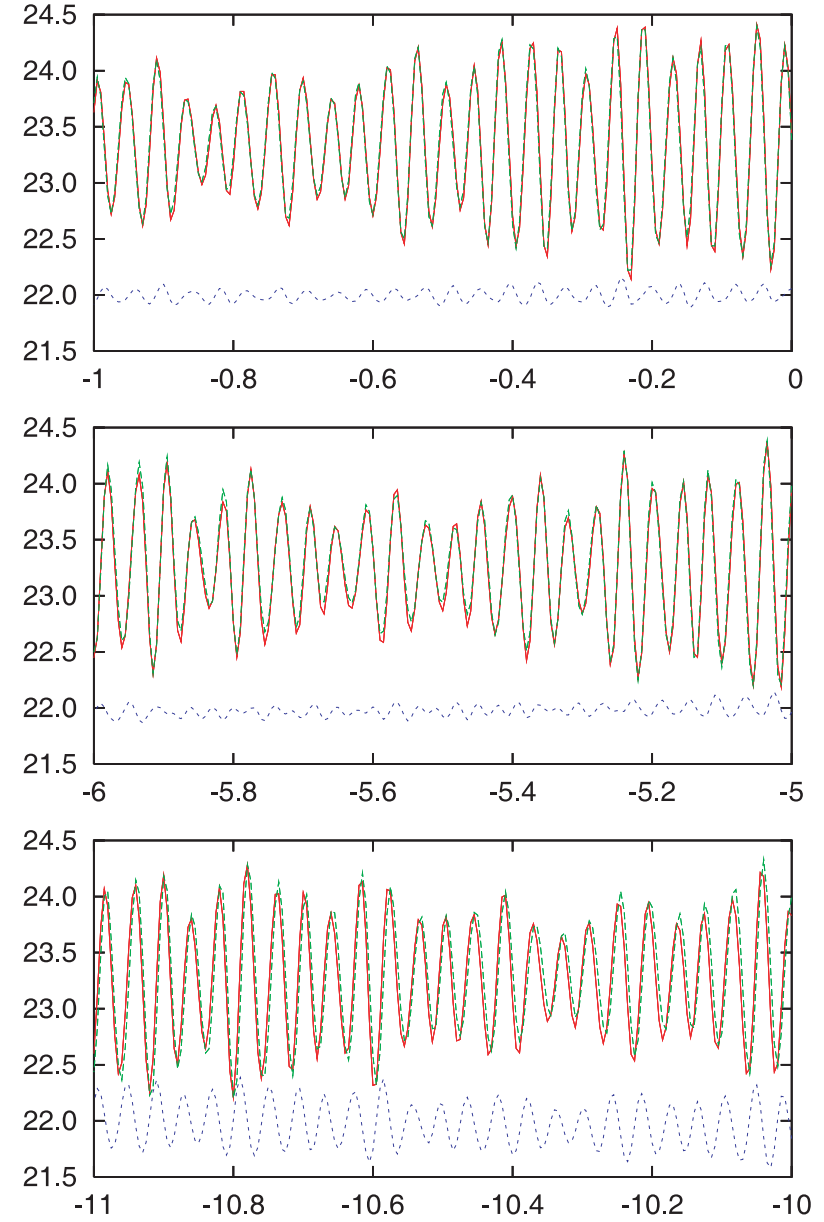

Fig. 15. Comparison of the solution of the obliquity La2004 (solid line) with its approximation using Table 7 (dotted line) with 26 periodic terms. The difference of two solutions $(+22$ degrees $)$ is also plotted.

evolution of the precession frequency. The equivalent formulas for the length of the day are (in hours)

$$
\begin{aligned}
& L O D^{-}=23.934468 \quad L O D^{+}=23.934468 \\
& \begin{array}{ll}
+7.432167 \mathrm{~T} & +7.444649 \mathrm{~T}
\end{array} \\
& -0.727046 T^{2} \quad-0.715049 T^{2} \text {. } \\
& +0.409572 T^{3} \quad+0.458097 T^{3} \\
& -0.589692 T^{4}
\end{aligned}
$$

One should note that Eqs. (39) and (41) correspond at the origin J2000 to a change in the semi major axis of the Moon of about $3.89 \mathrm{~cm} / \mathrm{yr}$, and a change of the LOD of $2.68 \mathrm{~ms} /$ century. These values slightly differ from the current ones of Dickey et al. (1994) $(3.82 \pm 0.07 \mathrm{~cm} / \mathrm{yr}$ for the receding of the Moon). This is understandable, as the Earth-Moon model in our integration that was adjusted to DE406 is simplified with respect to the one of DE406.

\section{Stability of the solution}

In such long term numerical computations, the sources of errors are the error of method due to the limitation of the numerical integration algorithm, the numerical roundoff error resulting from the limitation of the representation of the real numbers

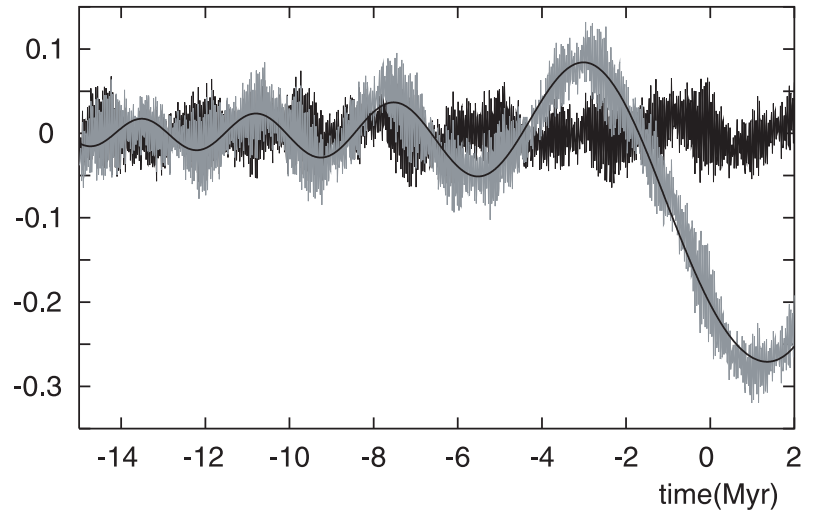

Fig. 16. Comparison of the solution of the precession La2004 with its approximation from -15 to $+2 \mathrm{Myr}$. The grey curve is obtained after removing uniquely the secular trend $\psi_{0}+p_{0} t+p_{1} t^{2}$. The dark curve are the residual (in radians) after removing the resonant term (Eq. (34)), which is also displayed in solid line.

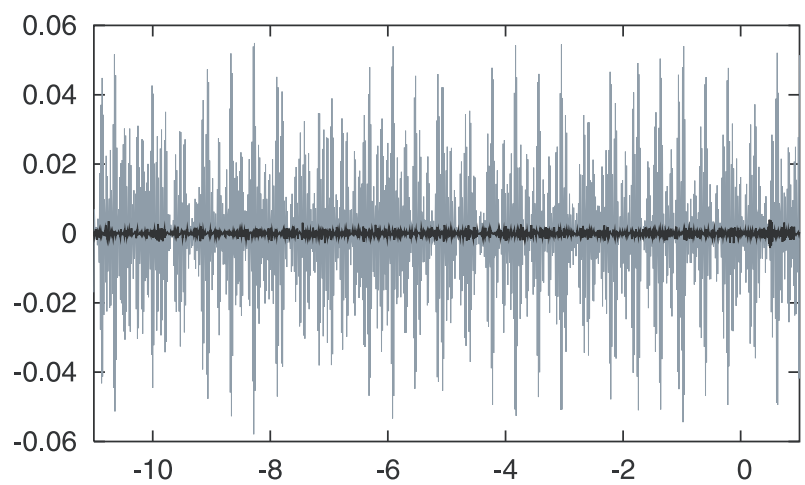

Fig. 17. Comparison of the solution of the climatic precession of La2004 with its approximation from -11 to +1 Myr. The grey curve is the full climatic precession $e \sin \bar{\omega}$. The dark curve are the residual after removing the approximation given by Eq. (37).

in the computer, and the errors of the initial conditions and of the model which are due to our imperfect knowledge of all the physical parameters in the Solar system, and to the necessary limitations of the model. Some of the main sources of uncertainty in the model for long term integrations were reviewed in (Laskar 1999). The effect of all these errors is amplified by the chaotic behaviour of the system, with an exponential increase of the difference between two solutions with different settings, until saturation due to the limited range of the considered variables. A summary of these limitations, extracted from (Laskar 1999) is given in Table 8, but it should be noted that these times of validity are probably pessimistic, as they correspond to analytical estimates based on the "worst case" situation. Our requirement for the precision of a long term solution is also not the same as for precise short term ephemeris. For the long term solutions, aimed at paleoclimate or qualitative studies, two solutions can be considered as similar, as long as the pattern of the two solutions (eccentricity for example), resulting (at first order) from the combination of various proper modes remain similar. This will last until some of the main proper modes get completely out of phase (see Sect. 7). It will be the same for the solutions in obliquity and precession. 

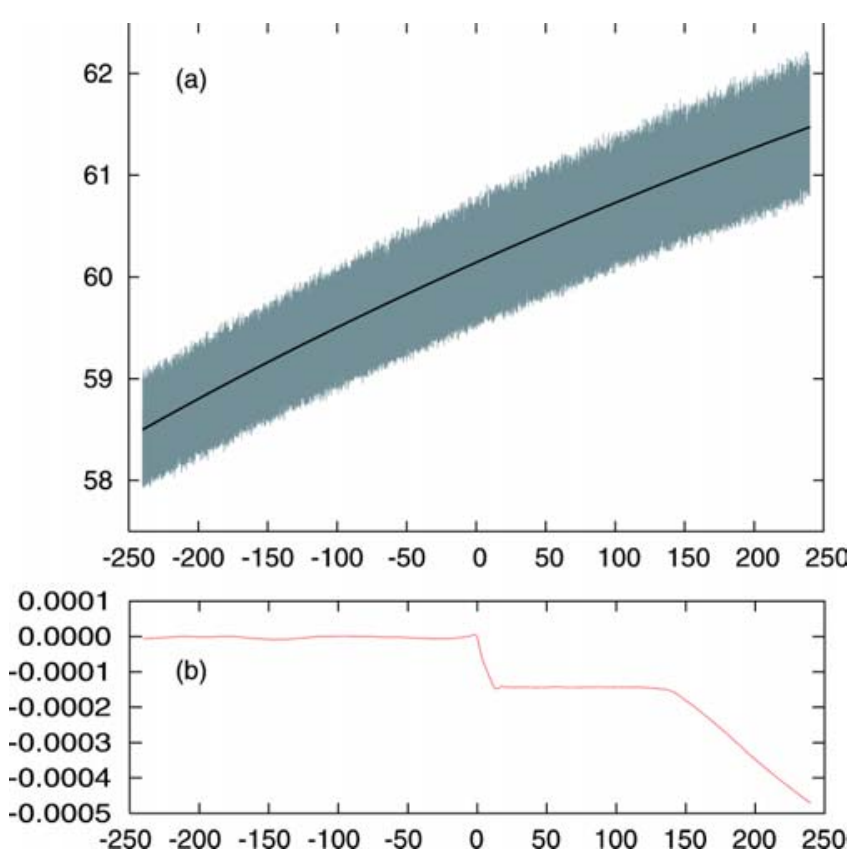

Fig. 18. a) Evolution of the Earth-Moon semi major axis (in Earth radii) from -250 to $+250 \mathrm{Myr}$. The grey zone is the result of the integration of the full equations, while the black curve is the integration of the averaged equations, as used in the precession computations; b) residuals with the fit of the averaged solution with the polynomial expression (39).
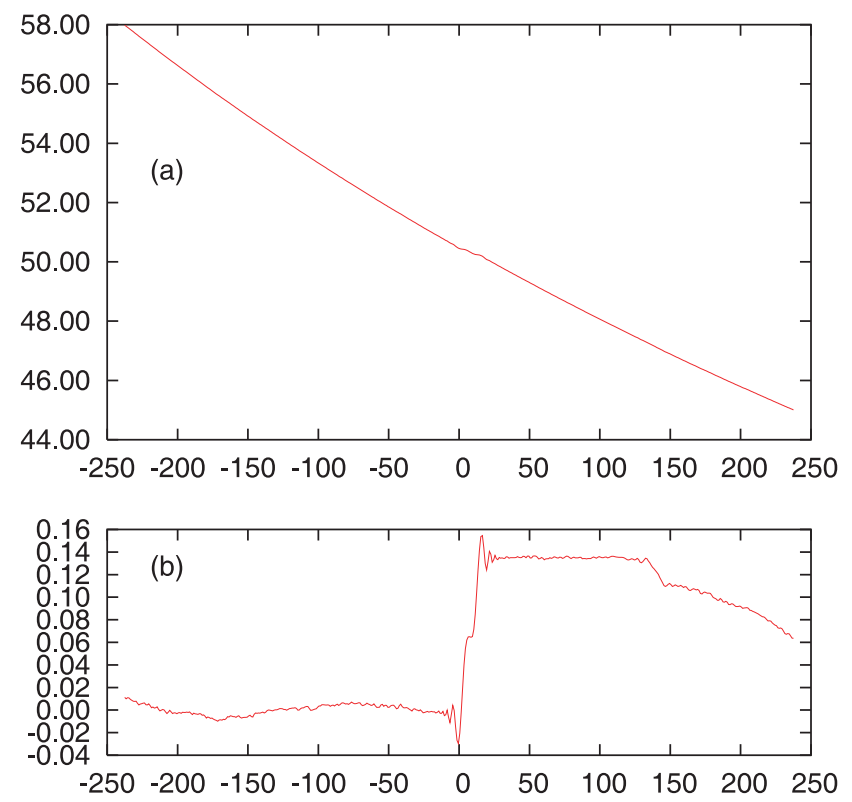

Fig. 19. a) Evolution of the precession frequency of the Earth $p$ (in $\operatorname{arcsec} / \mathrm{yr}$ ) -250 to $+250 \mathrm{Myr}$; b) residuals with the fit of the averaged solution with the polynomial expression (40).

\subsection{Orbital motion}

Practically, we have tested the stability of our solution by comparison of the eccentricity (Fig. 20) and inclination (Fig. 21) with different solutions. In Figs. 20a and 21a, the nominal solution La2004 (with stepsize $\tau=5 \times 10^{-3}$ years) is compared to an alternate solution, La2004*, with the same dynamical
Table 8. Main sources of uncertainty in the orbital solution (from Laskar 1999). For each limiting factor, an analytical estimate of the time of validity of the solution $T_{\mathrm{V}}$ is given (in Myr), taking into account the exponential growth of the error.

\begin{tabular}{lr}
\hline \hline Limiting factor & $T_{\mathrm{V}}$ \\
\hline Uncertainty on the masses and intial conditions & $38 \mathrm{Myr}$ \\
Contribution of the main Galilean satellites & $35 \mathrm{Myr}$ \\
Uncertainty in the Earth-Moon system evolution & $40 \mathrm{Myr}$ \\
Effect of the main asteroids & $32 \mathrm{Myr}$ \\
Mass loss of the Sun & $50 \mathrm{Myr}$ \\
Uncertainty of $2 \times 10^{-7}$ on the $J_{2}$ of the Sun & $26 \mathrm{Myr}$ \\
\hline
\end{tabular}
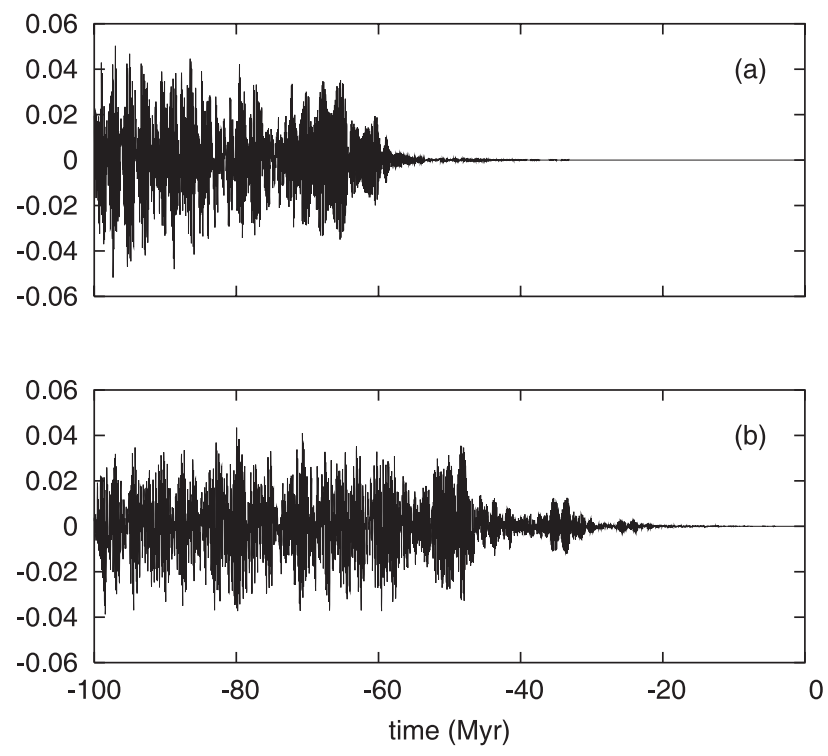

Fig. 20. Stability of the solution for eccentricity of the Earth. a) Difference of the nominal solution La2004 with stepsize $\tau=$ 5. $\times 10^{-3}$ years, and La2004*, obtained with $\tau^{*}=4.8828125 \times 10^{-3}$ years; $\mathbf{b})$ difference of the nominal solution with the solution obtained while setting $J_{2}^{S}=0$ for the Sun (instead of $2 \times 10^{-7}$ in the nominal solution).

model, and a very close stepsize $\tau^{*}=4.8828125 \times 10^{-3}$ years (Table 9). This special value was chosen in order that our output time span $h=1000$ years corresponds to an integer number (204 800) of steps, in order to avoid any interpolation problems in the check of the numerical accuracy. This is thus a test of the time of validity for obtaining a precise numerical solution, resulting from method and roundoff errors of the integrator. It is thus limited here to about $60 \mathrm{Myr}$.

This limitation of $60 \mathrm{Myr}$ is a limitation for the time of validity of an orbital solution, independently of the precision of the dynamical model. In order to go beyond this limit, the only way will be to increase the numerical accuracy of our computations, by improving the numerical algorithm, or with an extended precision for the number representation in the computer.

A second test is made on the uncertainty of the model. Several sources of uncertainty were listed in (Laskar 1999), and it was found that one of the main sources of uncertainty was due to the imprecise knowledge of the Solar 

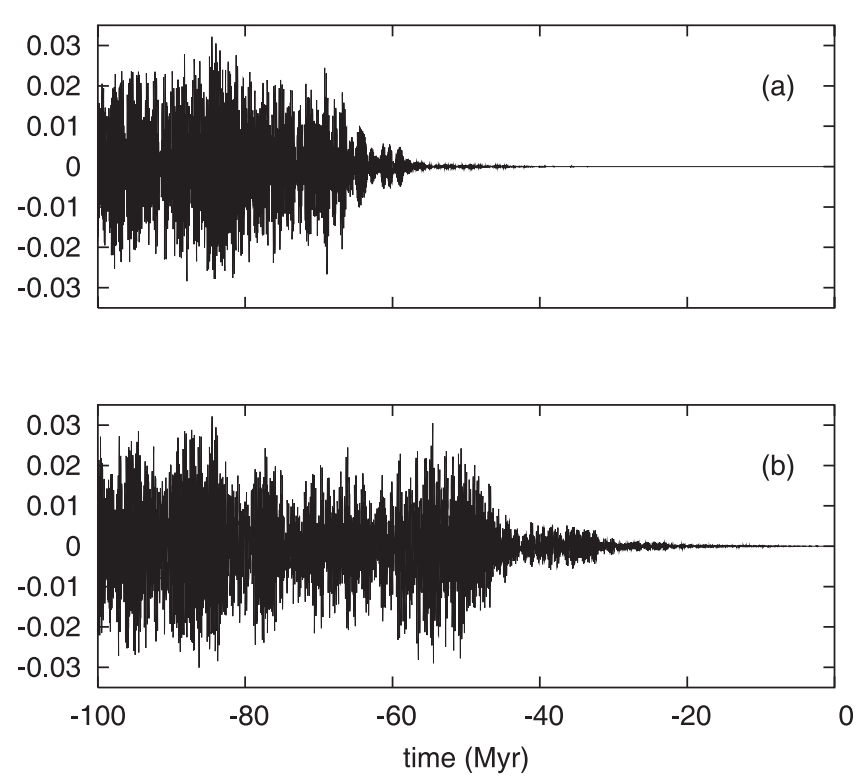

Fig. 21. Stability of the solution for inclination of the Earth. a) Difference of $\sin i / 2$ for the nominal solution La2004 with stepsize $\tau=5 . \times 10^{-3}$ years, and for La2004*, obtained with $\tau^{*}=$ $4.8828125 \times 10^{-3}$ years; b) difference of $\sin i / 2$ for the nominal solution with the solution obtained while setting $J_{2}^{\mathrm{S}}=0$ for the Sun (instead of $2 \times 10^{-7}$ in the nominal solution).

Table 9. Variants of the La2004 solutions. The nominal solution La2004 is obtained for a stepsize $\tau=5 \times 10^{-3}$, and a Solar oblateness $J_{2}^{\mathrm{S}}=2.0 \times 10^{-7}$. The other solutions, with different setting have been computed to test the stability of the nominal solution. In the last column $\gamma$ is the tidal dissipation factor.

\begin{tabular}{llcl}
\hline \hline Name & \multicolumn{1}{c}{$\tau(\mathrm{yr})$} & \multicolumn{1}{c}{$J_{2}^{\mathrm{S}}$} & $\gamma$ \\
\hline La2004 & $5 \times 10^{-3}$ & $2.0 \times 10^{-7}$ & 1 \\
La2004 $^{*}$ & $4.8828125 \times 10^{-3}$ & $2.0 \times 10^{-7}$ & 1 \\
La2004** $^{*}$ & $5.00000005 \times 10^{-3}$ & $2.0 \times 10^{-7}$ & 1 \\
La2004 $_{0}$ & $5 \times 10^{-3}$ & 0 & 1 \\
La2004 $_{1.0}$ & $5 \times 10^{-3}$ & $1.0 \times 10^{-7}$ & 1 \\
La2004 $_{1.5}$ & $5 \times 10^{-3}$ & $1.5 \times 10^{-7}$ & 1 \\
La2004 $^{0.95}$ & $5 \times 10^{-3}$ & $2.0 \times 10^{-7}$ & 0.95 \\
La2004 $^{0.90}$ & $5 \times 10^{-3}$ & $2.0 \times 10^{-7}$ & 0.90 \\
\hline
\end{tabular}

oblateness $\left(J_{2}^{S}\right)$ (Table 8). Its most recent determination, obtained with the SOHO and GONG helioseismic data give $J_{2}^{\mathrm{S}}=$ $(2.18 \pm 0.06) \times 10^{-7}$ (Pijpers 1998$)$, with a very similar value adopted in DE406 $\left(J_{2}^{\mathrm{S}}=2 \times 10^{-7}\right.$, Standish 1998), while in DE200, it was not taken into account (Newhall et al. 1983). We will thus consider that comparing the nominal solution La2004 (with $J_{2}^{\mathrm{S}}=2 \times 10^{-7}$ ) with an alternate solution (La2004 $)$ with $J_{2}^{\mathrm{S}}=0$ is representative of the uncertainty of the dynamical model for our long term integrations ${ }^{1}$ (Table 9).

The results La2004 - $\mathrm{La}_{2004_{0}}$ for the Earth's eccentricity and inclination are displayed in (Figs. 20b and 21b)

1 This empirical assumption is motivated by the fact that in Table 8 , an error of $2 \times 10^{-7}$ on $J_{2}^{\mathrm{S}}$ appear to be the largest limiting factor in the long term evolution of the solution. over 100 Myr. As for Mars (Laskar et al. 2004), the effect of $J_{2}^{\mathrm{S}}$ becomes noticeable after about $30 \mathrm{Myr}$ (26 Myr were predicted with an analytical estimate in (Laskar 1999), and the solution remains very similar over $40 \mathrm{Myr}$, and totally out of phase after $45 \mathrm{Myr}$. We will thus consider here that $40 \mathrm{Myr}$ is about the time of validity of our present orbital solution for the Earth. Other integrations made with $J_{2}^{\mathrm{S}}=1.5 \times 10^{-7}$ and $J_{2}^{S}=1.0 \times 10^{-7}$ (Table 9) gave practically the same estimates. It should be noted that with our present algorithms, we are much more limited by the precision of the model (Figs. 20b and 21b) than by the numerical accuracy (Figs. 20a and 21a).

\subsection{Obliquity and precession}

For the obliquity and precession, as was already mentioned in (Laskar et al. 1993), the main source of uncertainty is the evolution of the tidal dissipation and other related factors (see Sect. 4.1). Among these factors, one can mention the variations of the dynamical ellipticity of the Earth resulting from changes of the ice caps, or mantle convections. The maximal contribution of these effects can be estimated (see Levrard \& Laskar 2003), but it is very difficult to obtain at present a precise value of their contributions. Moreover, the largest dissipative term, resulting from body tides can evolve substantially as a result of the variation of the distribution of the continents, as most of the tidal dissipation occurs in shallow seas (Jeffreys 1920, 1976; Egbert \& Ray 2000).

In Fig. 22, we have explicited the result of a difference of $5 \%$ (Figs. 22a and c) and 10\% (Figs. 22b and d) in the tidal dissipation term of the Earth and the Moon ( $\Delta t$ and $\Delta t_{\mathrm{M}}$ in Table 1). With $5 \%$ error $\left(\mathrm{La} 2004^{(0.95)}\right)$, the solution of obliquity is valid over about $20 \mathrm{Myr}$, but with a $10 \%$ error $\left(\mathrm{La} 2004^{(0.90)}\right)$, the solution is out of phase after 20 Myr. Nevertheless, the uncertainty in the tidal dissipation appears mostly as a small change of the precession frequency $p$, which reveals in the obliquity solution as a time offset that does not change much the obliquity pattern. Indeed, in Figs. 22e and f, we have made a readjustment of the time scale for the solutions La2004 ${ }^{(0.95)}$ and $\mathrm{La} 2004^{(0.90)}$ of respectively

$\delta t_{(0.95)}=1.3346 \times 10^{-5} t-1.9362 \times 10^{-5} t^{2}$
$\delta t_{(0.90)}=1.4358 \times 10^{-5} t-3.9666 \times 10^{-5} t^{2}$

where $t$ is in Myr. With this new time scales, the different solutions become very close over $40 \mathrm{Myr}$, that is over the full range of validity of the orbital solution (Figs. 22e and f). An uncertainty of $10 \%$ in the tidal dissipation term thus corresponds mostly to an uncertainty in the timescale of $16 \mathrm{kyr}$ after $20 \mathrm{Myr}$, and $63 \mathrm{kyr}$ after $40 \mathrm{Myr}$.

\section{Resonant angles}

\subsection{Secular resonances}

Using the secular equations, Laskar (1990, 1992), demonstrated that the chaotic behavior of the Solar system arises from multiple secular resonances in the inner solar system. In particular, the critical argument associated to

$\theta=\left(s_{4}-s_{3}\right)-2\left(g_{4}-g_{3}\right)$ 

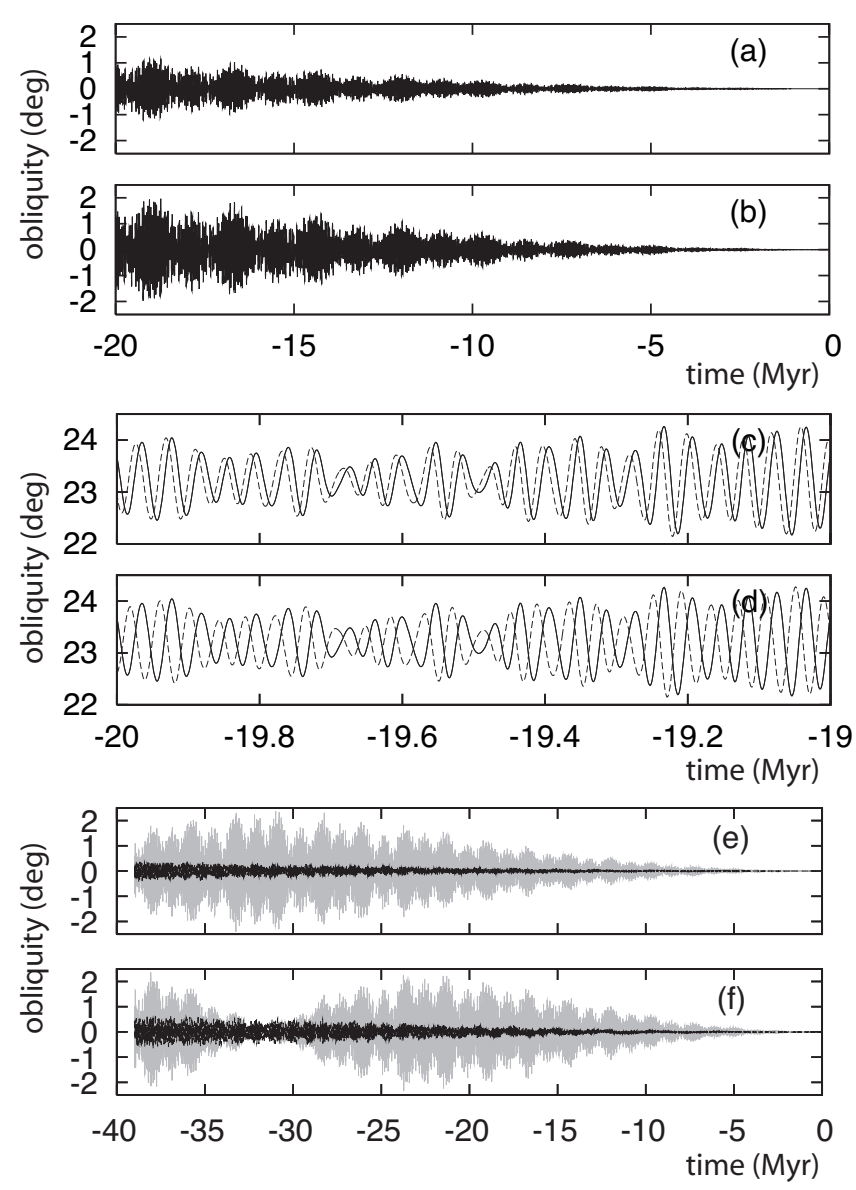

Fig. 22. Difference in obliquity for different tidal dissipation factor (Table 9): a) La2004 - La2004 ${ }^{(0.95)}$; b) La2004 - La2004 ${ }^{(0.90)}$. In c) and d), the nominal solution of the obliquity is plotted in solid line, while $\mathrm{La}_{2004^{(0.95)}}$ c) and $\mathrm{La} 2004^{(0.90)}$ d) are in dotted line. After an adjustment of the time scale, the differences of the solutions are much smaller over 40 Myr: e) La2004-La2004 ${ }^{(0.95)}$; f) La2004-La2004 ${ }^{(0.90)}$.

where $g_{3}, g_{4}$ are related to the precession of the perihelion of the Earth and Mars, $s_{3}, s_{4}$ are related to the precession of the node of the same planets, is presently in a librational state, but can evolve in a rotational state, and even move to libration in a new resonance, namely

$\left(s_{4}-s_{3}\right)-\left(g_{4}-g_{3}\right)=0$.

This reveals that a region of relatively strong instability results from the overlap (Chirikov 1979) of these two resonances. This result was questioned by Sussman \& Wisdom (1992), as they did not recover such a large variation for the secular frequencies, but reached only $2\left(s_{4}-s_{3}\right)-3\left(g_{4}-g_{3}\right)=0$ instead of Eq. (44). We have tested these resonant arguments in the nominal solution La2004, and in the alternate solutions La2004**, La2004 , La2004 $_{1.5}$ (Table 9).

In all variants of La2004 (Fig. 23 (top)), the resonant argument corresponding to $\theta=\left(s_{4}-s_{3}\right)-2\left(g_{4}-g_{3}\right)$ was in libration over the first $40 \mathrm{Myr}$, while the transition to circulation occurred at about $25 \mathrm{Myr}$ in La93. All the solutions La2004, La2004**, La2004 ${ }_{0}, \mathrm{La}_{2004_{1.5}}$ differ significantly beyond 40 to $50 \mathrm{Myr}$, but they all enter the $\left(s_{4}-s_{3}\right)-\left(g_{4}-\right.$ $\left.g_{3}\right)$ resonance within $100 \mathrm{Myr}$, comforting the conclusions of
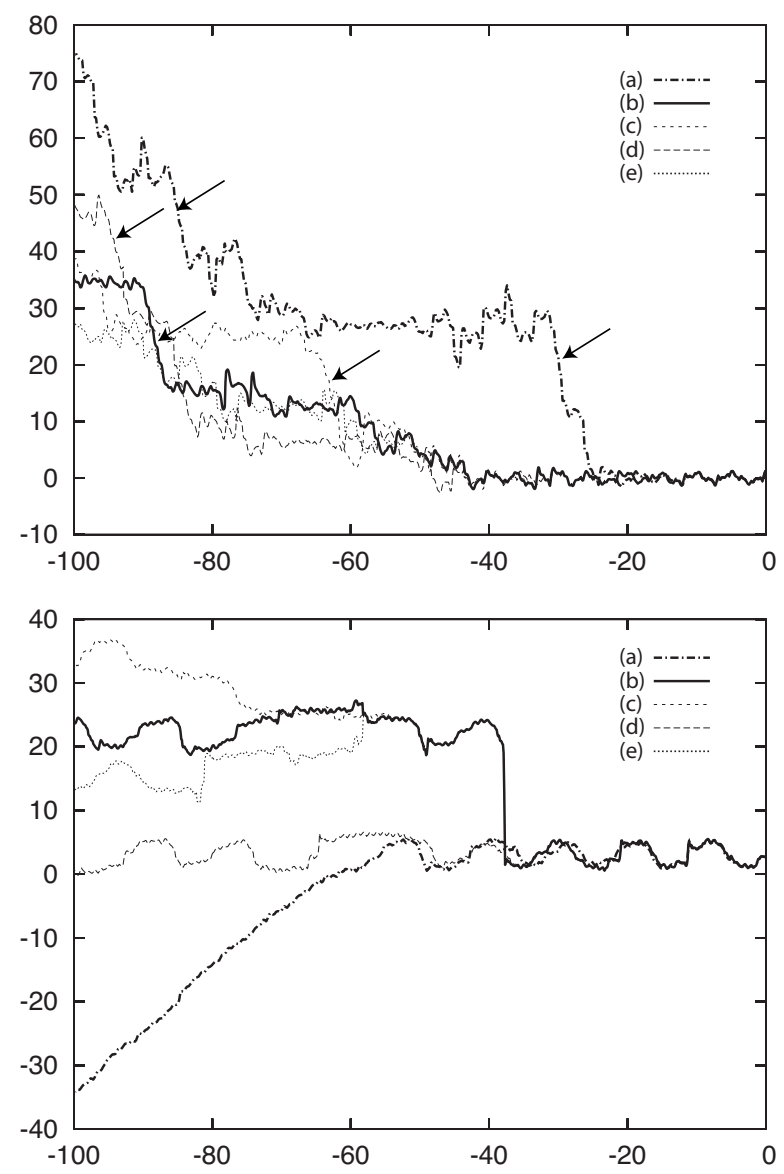

Fig. 23. Resonant arguments (in radians versus time in Myr) $\theta=$ $\left(s_{4}-s_{3}\right)-2\left(g_{4}-g_{3}\right)$ (top) and $\sigma=\left(g_{1}-g_{5}\right)-\left(s_{1}-s_{2}\right)$ (bottom) for different solutions: a) is the secular solution La93 (Laskar 1990; Laskar et al. 1993) while b) is the present nominal solution La2004. The characteristics of the other variants La2004 1.5 c), La2004 0 d), $\mathrm{La}_{2004^{* *}}$ e) are listed in Table 9. The arrows indicate the portions of the solutions when the argument $\left(s_{4}-s_{3}\right)-\left(g_{4}-g_{3}\right)$ is in libration.

(Laskar 1990, 1992). In Fig. 23 (top), the portions of the solutions where the argument of $\left(s_{4}-s_{3}\right)-\left(g_{4}-g_{3}\right)$ is in libration are pointed by arrows. It should be noted that at least over $250 \mathrm{Myr}$, the diffusion seems to remain confined between the resonances $\left(s_{4}-s_{3}\right)-2\left(g_{4}-g_{3}\right)$ and $\left(s_{4}-s_{3}\right)-\left(g_{4}-g_{3}\right)$. The other important resonant argument $\left(\left(g_{1}-g_{5}\right)-\left(s_{1}-s_{2}\right)\right)$ that was identified by Laskar $(1990,1992)$ as the origin of the chaotic behavior of the inner planets presents in all the variations of the numerical solutions La2004, La2004**, La2004, La2004 $_{1.5}$ a similar behavior as for the secular solution La93 (Fig. 23 (bottom)).

\subsection{Geological evidence of chaos}

The date of the first transition from libration to circulation of the resonant argument related to $\theta=\left(s_{4}-s_{3}\right)-2\left(g_{4}-g_{3}\right)$ is a macroscopic feature displaying the chaotic diffusion of the planetary orbits. It is thus very tempting to search whether this feature could be observed in the geological data that extend now in continuous records beyond $30 \mathrm{Myr}$. 
The direct observation of the individual arguments related to $g_{3}, g_{4}, s_{3}, s_{4}$ is certainly out of reach. But it may be possible to follow the evolution in time of the differences $g_{3}-g_{4}$ and $s_{3}-s_{4}$. Indeed, $s_{3}-s_{4}$ appears as a beat of about 1.2 million of years in the solution of obliquity, as the result of the beat between the $p+s_{3}$ and $p+s_{4}$ components of the obliquity, where $p$ is the precession frequency of the axis (see Laskar 2004 , Fig. 7). In a similar way, $g_{3}-g_{4}$ appears as a beat with period of about 2.4 Myr in the climatic precession. One understands that because of the occurrence of these beats, the detection of the resonant state in the geological data can be possible, as one has to search now for phenomena of large amplitude in the geological signal. Indeed, in the newly collected data from Ocean Drilling Program Site 926, the modulation of 1.2 Myr of the obliquity appears clearly in the spectral analysis of the paleoclimate record (Zachos et al. 2001). Moreover, using the ODP legs 154 and 199, Pälike et al. (2004) could find some evidence that the critical argument of $\theta$ did not show a transition to circulation at $25 \mathrm{Myr}$, as in La93, but remained in libration over $40 \mathrm{Myr}$, as in the present new solution La2004. This result is very impressive, although the search for the determination of these resonant angles in the geological data is just starting. We may expect that a careful analysis, and new data spanning the most recent 60 to $100 \mathrm{Myr}$, will allow to determine the possible succession of resonant states in the past, allowing, for example, to discriminate between the solutions displayed in Fig. 23. Of particular importance would be the detection of the first transition from libration to circulation of the resonant argument $\left(s_{4}-s_{3}\right)-2\left(g_{4}-g_{3}\right)$. This program, if completed, will provide some extreme constraint for the gravitational model of the Solar System. Indeed, the observation of a characteristic feature of the solution at 40 to $100 \mathrm{Myr}$ in the past, because of the exponential divergence of the solutions, will provide a constraint of $10^{-4}$ to $10^{-10}$ on the dynamical model of the Solar System.

\section{Mesozoic timescale}

An astronomically calibrated time scale for geological data has been achieved successfully over the Neogene period (0-23.03 Myr) (Lourens et al. 2004), and the next challenge will be to improve the astronomical precision of the solution, in order to extend this over the Paleogene (23 to $65 \mathrm{Myr}$ ). Beyond this time length, it is hopeless to expect to obtain a precise solution for the orbital elements of the Earth. On the other hand, some features of the solution have a longer time of stability, if they are related to the outer planets secular frequencies (Laskar 1990).

A very important period present in geological records is the $405 \mathrm{kyr}$ period related to the $g_{2}-g_{5}$ argument, that is the leading term in the solution of eccentricity (Table 6). In Fig. 24, we have tested the stability of this argument over the full period of our integrations, that is over $250 \mathrm{Myr}$, that extents over the Mesozoic geological era (about 65 to $250 \mathrm{Myr}$ ). First, we have compared the argument related to $g_{5}$ over 3 variants (La2004*, $\mathrm{La} 2004_{0}, \mathrm{La}_{2004} .5$ ) of La2004, that reflect both the numerical errors and the errors of the model. This argument, related to Jupiter and Saturn is extremely stable, and does not show
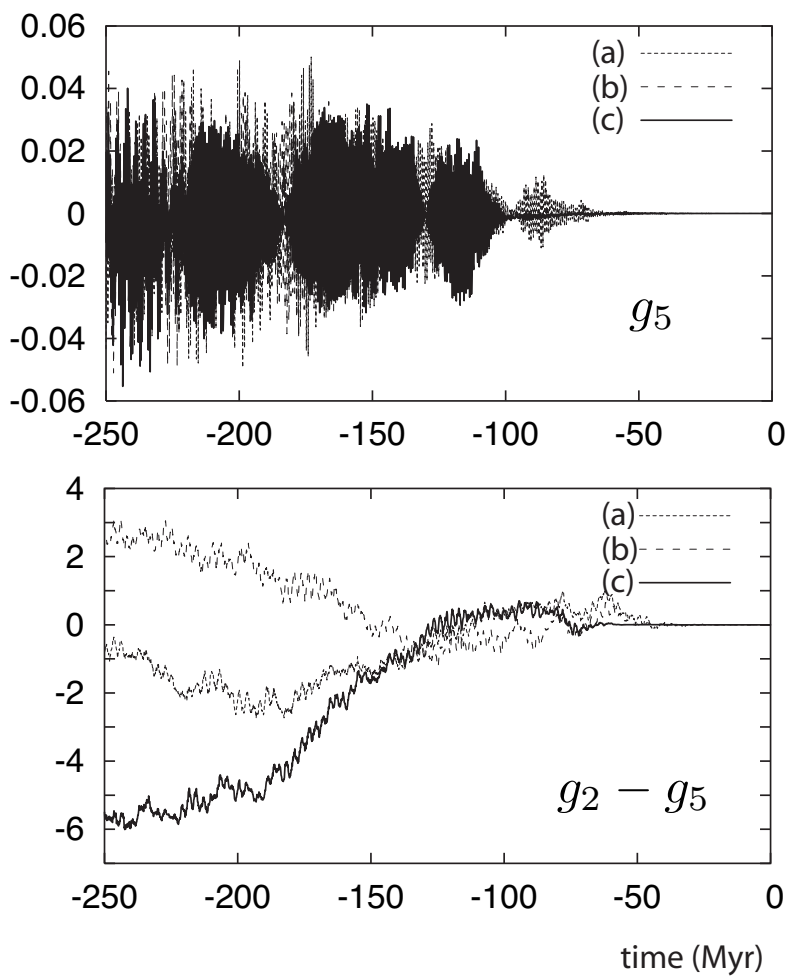

Fig. 24. Stability of the $g_{5}$ (top) and $g_{2}-g_{5}$ (bottom) arguments. The difference (in radians) of the angles related to $g_{5}$ and $g_{2}-g_{5}$ from the nominal solution La2004 and an alternate solution La2004 ${ }_{1.5}$ a),

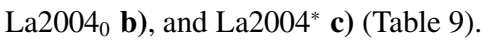

variations of more than 0.05 radians over the full $250 \mathrm{Myr}$ period (Fig. 24 (top)). In Fig. 24 (bottom), we have plotted the same quantities for $g_{2}-g_{5}$. The dispersions over 50, 100, and $250 \mathrm{Myr}$ are about 0.3, 1.0, 6.2 radians, that correspond to absolute error in time respectively of about 20, 70, and $400 \mathrm{kyr}$ over the same periods.

It is thus possible to use this argument, that is visible in many sedimentary records of the Early Mesozoic (Olsen \& Kent 1999, and references therein) as an absolute geological time scale over the whole Mesozoic period. In order to facilitate the establishment of this timescale, we have searched for a best fit or the period of this argument over all the solutions of Table 9. Within the uncertainty of this procedure, the best value for the frequency $g_{2}-g_{5}$ is

$v_{405}=3.200^{\prime \prime} / \mathrm{yr}$

which corresponds exactly to a period

$P_{405}=405000 \mathrm{yr}$

This value differs by less than $0.001^{\prime \prime} / \mathrm{yr}$ from the same quantity from Table 6 , where the direct output of the frequency analysis is given. We propose here that in the establishment of geological timescales, the value $3.200^{\prime \prime} / \mathrm{yr}$ should be used. The maximum error over 50, 100, 200, and $250 \mathrm{Myr}$ should then be less than respectively $30,150,350$, and $500 \mathrm{kyr}$. The $405 \mathrm{kyr}$ term is the largest term of the eccentricity solution. In Fig. 26, the eccentricity of the Earth from La2004, filtered in the 


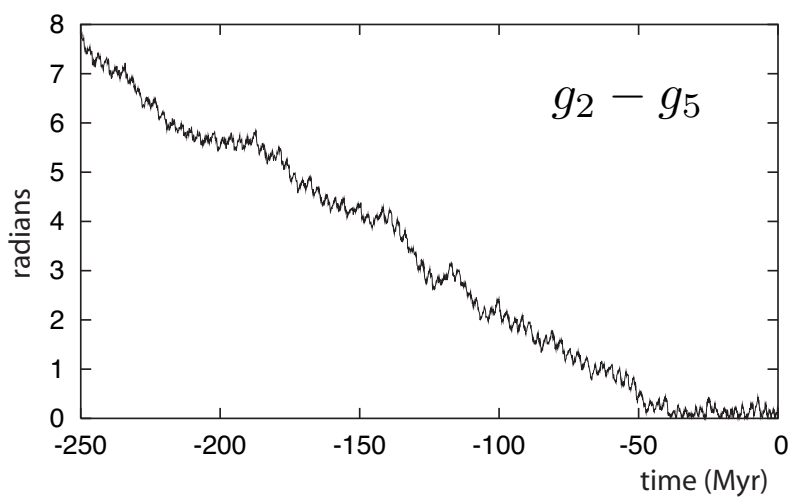

Fig. 25. $405 \mathrm{kyr}$ term in eccentricity. Maximum difference (in radians) of the argument $\theta_{g_{2}-g_{5}}$ of $g_{2}-g_{5}$ in all 6 solutions of Table 9 (La2004, $\mathrm{La}_{2004}{ }, \mathrm{La}_{2004}^{* *}, \mathrm{La} 2004_{0}, \mathrm{La}_{2004_{1.0}}, \mathrm{La}_{2004_{1.5}}$ ) with respect to the linear approximation $\theta_{g_{2}-g_{5}}=2434^{\prime \prime}+3.200^{\prime \prime} t$ where $t$ is in yr.
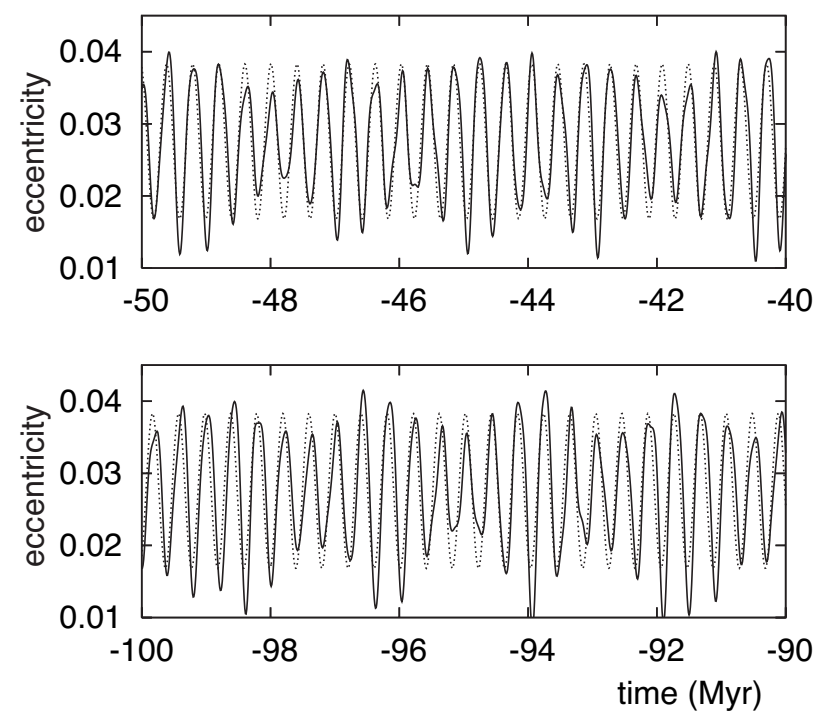

Fig. 26. Eccentricity of the Earth. Nominal solution La2004 filtered in the interval $\left[2.2^{\prime \prime} / \mathrm{yr}, 8^{\prime \prime} / \mathrm{yr}\right]$ (solid line), and approximation with a single periodic term (47) (dotted line).

interval $\left[2.2^{\prime \prime} / \mathrm{yr}, 8^{\prime \prime} / \mathrm{yr}\right]$, is plotted, as well as a solution for the eccentricity, limited to the single $405 \mathrm{Kyr}$ term

$e_{405}=0.027558-0.010739 \cos \left(2434^{\prime \prime}+3.200^{\prime \prime} t\right)$

where $t$ is in yr. As it is apparent in the figure, the agreement between the two solutions is extremely good over $100 \mathrm{Myr}$. Beyond that time, the various possible solutions will become out of phase, within the uncertainty of Fig. 25. One should note that the initial phase at the origin (2434 arcsec) corresponds to an offset of only $760 \mathrm{yr}$, so in most cases it can be neglected.

\section{Discussion and future work}

\subsection{Discussion}

The new orbital solution of the Earth that is presented here can be used for paleoclimate computations over 40 to 50 Myr. Beyond that time interval, the precision of the solution cannot be guaranteed but we nevertheless provide the solution over $100 \mathrm{Myr}$ on our Web site www . imcce . fr/Equipes/ASD/insola/earth/earth.html as reference, and for a possible use, with caution, over the full Paleogene period (up to 65 Myr). As in Laskar et al. (1993), the solution for precession and obliquity is not as accurate as the orbital solution. This is mainly due to the uncertainty that remains in the tidal dissipation in the Earth-Moon system. We expect the present solution to be precise over about $20 \mathrm{Myr}$ (see Sect. 9.2), but additional work is certainly needed here.

When taking into account the dissipation in the EarthMoon system, it is remarkable that in the time interval [-250 Myr, +250 Myr], the only major event in the obliquity of the Earth occurs in the vicinity of the present time (Fig. 14). We have discarded the possibility of some numerical artefact by integrating different models. In particular, we find the same slow decrease of about 0.38 degrees in the obliquity over about $20 \mathrm{Myr}$ either when we integrate the obliquity and precession equations of Sect. 4 at the same time as the orbital equations, or when the precession and obliquity are computed afterwards, with a forced orbital motion. Moreover, the behavior of the solution is perfectly explained by the crossing of the $s_{6}+g_{5}-g_{6}$ resonance, as it is explicited in full details in the appendix.

The crossing of this resonance will occur in the next $20 \mathrm{Myr}$, but one could wonder whether this crossing could have also occured in the recent past and thus had a possible impact on the past Earth climate.

In (Laskar et al. 1993), we searched for the possibility to encounter this resonance in the past by changing the momentum of inertia of the Earth as a result of the ice load changes occuring during an ice age, but it seems that our values for the change of momentum of inertia during an ice age was overestimated (Mitrovica \& Forte 1995; Levrard \& Laskar 2003). In a similar way, Forte \& Mitrovica (1997) have advocated the mantle convection as a mean to change the values of the momentum of inertia of the Earth in the past, and thus to eventually cross the $s_{6}+g_{5}-g_{6}$ resonance in the past. Their results are very sensitive to their models, and it is not clear that the induce change of momentum of inertia will be able to counteract the effect of tidal dissipation that drives the obliquity away from the resonance in the past. Moreover, the analysis of geological data in the past few Myr (Lourens et al. 2001; Pälike \& Shackleton 2001) tends to show that the precession frequency has actually increased in the past and thus, that the system was driven away from the resonance. Additional studies on this topic are certainly welcome, and we should still search for the possibility of a crossing of the $s_{6}+g_{5}-g_{6}$ resonance in the past, as it may have impacted the climatic history of the Earth, but in absence of new evidence, we should consider that the proximity of this resonance is pure coincidence.

\subsection{Future work}

The next step for the improvement of the solutions will be to obtain an accurate orbital solution over the full Paleogene period (65 Myr). The numerical accuracy of our integrator 
already fulfills this requirement (Fig. 23), although we expect to slightly improve it by switching to extended computer precision.

The improvement of the model and parameters is much more difficult, and will require additional work. First, we will have to include in our model supplementary contributions in order to compare much more closely to DE406. In particular, in the present work, to save computer time, we had to simplify the interactions in the Earth-Moon system. In the next integration, we will take into account additional contributions in the body body interactions of the Earth-Moon system, and also the libration of the Moon. We will also have to adapt the tidal model in the Earth-Moon system. We will need to take into account the effect of the 5 main asteroids that are present in DE406, and probably derive a small model for the contribution of the 295 additional asteroids that are considered in DE406. Indeed, the consideration of the main asteroids appears as the next most important source of uncertainty for the long term solution (Table 8). Some of the other factors listed in Table 8 can easily be taken into account, as the consideration of the main Galilean satellites, or the mass loss of the Sun.

Finally, it will also be important to reestablish the precision of the parameter of the model by a direct comparison to the observations. For this part, we expect to benefit from the effort that is developed at present in our laboratory for the improvement of short time ephemeris devoted to the reduction of Earth based and space missions observations (Fienga \& Simon 2004).

The fulfilment of this program will allow to improve on the main limiting factors listed in Table 8. It should be stressed again here that the limiting times listed in Table 8 are somewhat pessimistic. We can thus hope that after completing this effort, the orbital solution may be valid over 50 to $65 \mathrm{Myr}$.

Nevertheless, although this program may lead to a solution valid over about $60 \mathrm{Myr}$, obtaining a precise solution for the precession and obliquity of the Earth's axis over $65 \mathrm{Myr}$ is much more difficult, as it will require a modelization of the evolution of the various dissipative contributions (see Sect. 4.1) over this period. An alternate way will be to fit the evolution of the main precession frequency to the geological data over the Paleogene period. Although some results have been already obtained in this direction over some shorter time span (Lourens et al. 2001; Pälike \& Shackleton 2001), to extract the variation of the precession frequency from paleoclimate stratigraphic data is still a challenge, but we hope that the present paper will provide a good basis for this future work.

\section{Appendix A: Fresnel integrals and resonances}

In this section, we show that Fresnel integrals are very good approximations for the crossing of the resonance. Let us define the Fresnel integrals (we have dropped here the usual $\pi / 2$ factor) as

$C(x)=\int_{0}^{x} \cos \left(t^{2}\right) \mathrm{d} t ; \quad S(x)=\int_{0}^{x} \sin \left(t^{2}\right) \mathrm{d} t$.

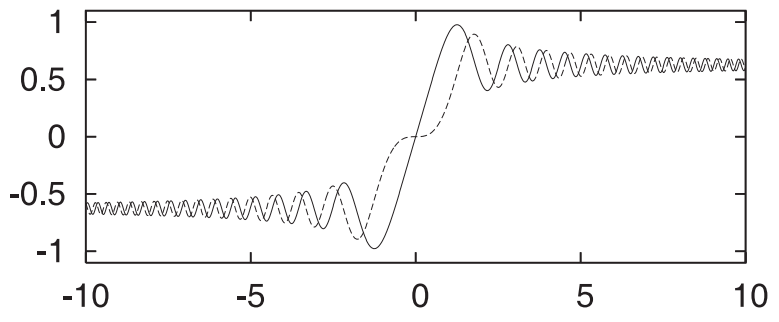

Fig. A.1. Fresnel functions $C(x)$ (solid line) and $S(x)$ (dotted line).

We have the expansions, for $x \geq 0$ (Abramowitz \& Stegun 1965)

$C(x)=\frac{1}{2} \sqrt{\frac{\pi}{2}}-P(x) \cos x^{2}+Q(x) \sin x^{2}$

$S(x)=\frac{1}{2} \sqrt{\frac{\pi}{2}}-P(x) \cos x^{2}-Q(x) \sin x^{2}$

with the asymptotic expansions

$P(x) \simeq \frac{1}{2}\left(\frac{1}{2 x^{3}}-\frac{1.3 .5}{2^{3} x^{7}}+\cdots\right)$
$Q(x) \simeq \frac{1}{2}\left(\frac{1}{x}-\frac{1}{2^{2} x^{5}}+\frac{1.3 .5 .7}{2^{4} x^{9}}+\cdots\right)$.

We thus have a jump from the asymptotic value at $-\infty$ to the value at $+\infty$ of $\sqrt{\pi / 2} \approx 1.253$ (Fig. A.1). Due to tidal dissipation, the precession angle evolves in first approximation as Eq. (34)

$\psi=\psi_{0}+p_{0} T+p_{1} T^{2}$

with this evolution, a term of frequency $v_{k}+p$ in Eq. (30) may cross the resonance. In particular, we can compute the contribution of the term $v_{23}$ in the quasiperiodic decomposition of $\zeta$ (Table 5). We will have from Eq. (28),

$\left.\frac{\mathrm{d} \varepsilon}{\mathrm{d} t}\right|_{23}=-2 a_{23} v_{23} \sin \left(\left(v_{23}+p_{0}\right) t+p_{1} t^{2}+\phi_{23}+\psi_{0}\right)$.

More generally, for $a>0$, the integral of

$\sin \left(a t^{2}+b t+c\right)$

is

$\frac{1}{\sqrt{a}}\left(\cos (\delta) S\left(\left(x+\frac{b}{2 a}\right) \sqrt{a}\right)+\sin (\delta) C\left(\left(x+\frac{b}{2 a}\right) \sqrt{a}\right)\right)$,

where $\delta=c-b^{2} / 4 a$, and the asymptotic offset $\Delta^{(\varepsilon)}$ due to the crossing of the resonance of the term (A.6) is easily obtained as

$\Delta^{(\varepsilon)}=\sqrt{\frac{\pi}{a}} \cos \left(\delta-\frac{\pi}{4}\right)$.

With the data for the resonant term $v_{23}$, we have

$a=p_{1} ; \quad b=v_{23}+p_{0} ; \quad c=\phi_{23}+\psi_{0}$, 


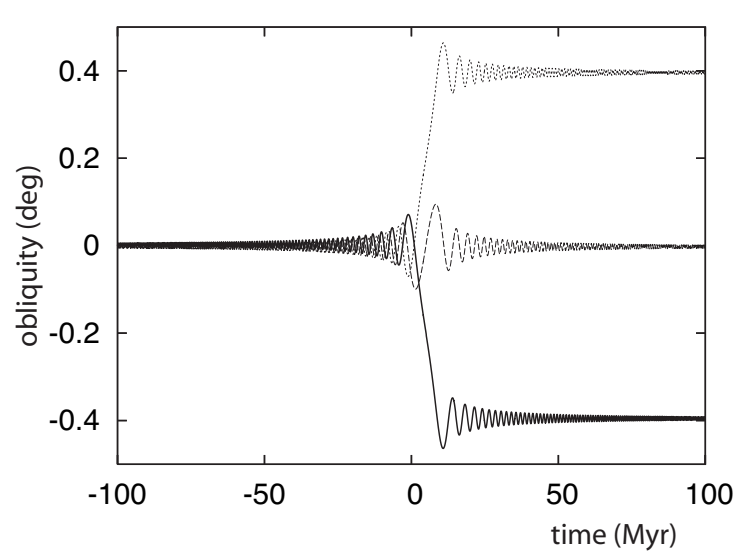

Fig. A.2. Obliquity variation (in degree) versus time resulting from the crossing of the resonance in the $v_{23}$ term (solid line). In the same plot, the dependence of the precession phase is illustrated. for $\delta=-\pi / 4$ the obliquity offset is null (dashed line) while it is maximal for $\delta=+\pi / 4$. The nominal solution (solid line) corresponds nearly to a maximal decrease of the obliquity.

and as the term is of amplitude $-2 a_{23} v_{23}$, we obtain (Fig. A.2) an offset of amplitude

$\Delta_{23}^{(\varepsilon)} \approx-0.398046 \mathrm{deg}$

that is very close to the value 0.377602 deg obtained in Sect. 8.3 for the complete integration. It should be noted that the amplitude of the offset depends critically of the phase $c=\phi_{23}+\psi_{0}$. Indeed, a maximum amplitude for the offset will be obtained when $\delta=\pi / 4 \bmod (\pi)$, which leads to

$\left|\Delta_{23}^{(\varepsilon)}\right|_{\max }=2 a_{23} v_{23} \sqrt{\pi / p_{1}} \approx 0.398721 \mathrm{deg}$.

while no offset will occur when $\delta=-\pi / 4 \bmod (\pi)$. In the present case, the actual value of the offset is thus close to its maximum possible value. One should also note that apart from the drift that occurs at the resonance, which ranges from about -0.4 to +0.4 degree, the maximum oscillation of the obliquity is of about 0.1 degree (Fig. A.2).

Acknowledgements. J.L. thanks J. Landwehr for providing a copy of Pilgrim's work, A. Vernekar for a copy of his monograph, and H. Pälike for his comments. This work benefited from support from INSU-CNRS, PNP-CNRS, and CS, Paris Observatory.

\section{References}

Adhémar, J. 1842, Révolutions de la mer, (Paris: Bachelier)

Agassiz, L. 1840, Études sur les glaciers (Paris)

Arnold, V. I., Kozlov, V. V., \& Neishtadt, A. I. 1988, Mathematical aspects of classical and celestial mechanics, Dyn. Systems III, ed. V. I. Arnold (New York: Springer)

Berger, A. L. 1978, J. Atmos. Sci., 35, 2362

Bills, B. G. 1994, Geophys. Res. Lett., 21(3), 177

Bretagnon, P. 1974, A\&A, 30, 141

Bretagnon, P. 1984, Celest. Mech., 34, 193

Bretagnon, P., \& Simon, J.-L. 1990, A\&A, 239, 387

Brouwer, D., \& Van Woerkom, A. J. J. 1950, Astron. Papers Am. Ephem., XIII, Part II, 81

Chapman, S., \& Lindzen, R. S. 1970, Atmospheric tides (Dordrecht: D. Reidel)
Chirikov, B. 1979, Phys. Rep., 52, 263

Correia, A., \& Laskar, J. 2003, J. Geophys. Res., 108(E11), 5123

Correia, A., Laskar, J., \& Néron de Surgy, O. 2003, Icarus, 163, 1

Croll, J. 1890, Climate and Time (London)

Darwin, G. H. 1880, Philos. Trans. R. Soc. London, 171, 713

Dickey, J. O., Bender, P. L., Faller, et al. 1994, Science, 265, 182

Duriez, L. 1977, A\&A, 54, 93

Egbert, G. D., \& Ray, R. D. 2000, Nature, 405, 775

Fienga, A., \& Simon, J.-L. 2004, submitted

Forte, A., \& Mitrovica, J. X. 1997, Nature, 390, 676

Gradstein, F., Ogg, J., \& Smith, A. 2004, A Geologic Time Scale 2004 (UK: Cambridge University Press), in press

Haretu, S. C. 1885, Annales de l'Observatoire de Paris, 18, I1

Harzer, P. 1895, Die Säcularen veränderungen der bahnen der grossen planeten, Fürstlich Jablonowski'schen Gesellschaft, Leipzig

Hays, J. D., Imbrie, J., \& Schackleton, N. J. 1976, Science, 194, 1121

Hill, G. 1897, AJ, 17(11), 81

Imbrie, J. 1982, Icarus, 50, 408

Imbrie, J., \& Imbrie, K. P. 1979, Ice Ages: Solving the Mystery (MA: Harvard University Press)

Ito, T., \& Tanikawa, K. 2002, MNRAS, 336, 483

Ito, T., Masuda, K., Hamano, Y., \& Matsui, T. 1995, J. Geophys. Res., 100,15147

Jeffreys, H. 1920, Phil. Trans. R. Soc. London, Ser. A, 221, 239

Jeffreys, H. 1976, The Earth, Its Origin, History, and Physical Constitution, 6th ed. (Cambridge, UK: Cambridge University Press)

Kinoshita, H. 1977, Cel. Mech., 15, 277

Lagrange, J. L. 1774, Oeuvres complètes, t. IV, Gauthier-Villars, Paris, 1869,111

Lagrange, J. L. 1776, Oeuvres complètes, t. IV, Gauthier-Villars, Paris, 1869,255

Lagrange, J. L. 1777, Oeuvres complètes, t. VI, Gauthier-Villars, Paris, 1873, 633

Lagrange, J. L. 1781, Oeuvres complètes, t. V, Gauthier-Villars, Paris, 1870,125

Lagrange, J. L. 1782, Oeuvres complètes, t. V, Gauthier-Villars, Paris, 1870,211

Lambeck, K. 1980, The Earth's variable rotation (UK: Cambridge University Press)

Lambeck, K. 1980, Geophysical Geodesy (UK: Oxford University Press)

Laplace, P. S. 1773-1776, Oeuvres complètes, t. VIII, GauthierVillars, Paris, 1891, 199

Laplace, P. S. 1776, Oeuvres complètes, t. VIII, Gauthier-Villars, Paris, 1891, 369

Laskar, J. 1984, Théorie générale planétaire: Eléments orbitaux des planètes sur 1 million d'années, Thèse, Observatoire de Paris

Laskar, J. 1985, A\&A, 144, 133

Laskar, J. 1986, A\&A, 157, 59

Laskar, J. 1988, A\&A, 198, 341

Laskar, J. 1989, Nature, 338, 237

Laskar, J. 1990, Icarus, 88, 266

Laskar, J. 1992, in Chaos, resonance and collective dynamical phenomena in the solar system, Ferraz-Mello, S. (1) (Kluwer Acad. Publ.), IAU Symp., 152

Laskar, J. 1999, Phil. Trans. R. Soc. Lond. A., 357, 1735

Laskar, J. 1999, Introduction to frequency map analysis, in NATO ASI 533 3DHAM95, S'Agaro, Spain, C. Simò (Kluwer), 134

Laskar, J. : 2001, Astronomical Solutions for Paleoclimate Studies,. AGU Fall Meeting 2001 Abstracts U11A-01

Laskar, J. 2003, Ann. Henri Poincaré, 4, Suppl. 2, S693

Laskar, J. 2003, in Hamiltonian systems and Fourier analysis, ed. Benest et al. (Taylor, Francis), in press 
Laskar, J., Joutel, F., \& Boudin, F. 1993, A\&A, 270, 522

Laskar, J., \& Robutel, P. 2001, Celest. Mech., 80, 39

Laskar, J., Correia, A. C. M., Gastineau, M., et al. 2004, Icarus, 170, 343

Le Verrier, U. 1856, Ann. Obs. Paris, II, Mallet-Bachelet, Paris

Levrard, B., \& Laskar, J. 2003, Journal Geophys. Int., 154, 970

Lourens, L. J., Wehausen, R., \& Brumsack, H. J. 2001, Nature, 409, 1029

Lourens, L. J., Hilgen, F. J., Shackleton, N. J., Laskar, J., \& Wilson, D. 2004, in The Neogene Period, ed. F. Gradstein, J. Ogg, \& A. Smith, A Geologic Time Scale 2004 (UK: Cambridge University Press), in press

Lumb, L. I., \& Aldridge, K. D. 1991, J. Geomagn. Geoelectr., 43, 93

MacCarthy, D. D. 1992, IERS Standards, IERS Technical note 13, Observatoire de Paris

MacCarthy, D. D., \& Petit, G. 2004, IERS Standards, IERS Technical note 13, Observatoire de Paris

Mignard, F. 1979, The Moon and the Planets, 20, 301

Mignard, F. 1980, The Moon and the Planets, 23, 185

Mignard, F. 1981, The Moon and the Planets, 24, 189

Milani, A., Nobili, A. N., \& Carpino, M. 1987, A\&A, 172, 265

Milankovitch, M. 1941, Kanon der Erdbestrahlung und seine Andwendung auf das Eiszeitenproblem, Royal Serbian Academy

Mitrovica, J. X., \& Forte, A. 1995, Geophys. J. Int., 121, 21

Néron de Surgy, O., \& Laskar, J. 1997, A\&A, 318, 975

Newhall, X. X., Standish, E. M., \& Williams, J. G. 1983, A\&A, 125, 150

Olsen, P., \& Kent, D. 1999, Phil. Trans. R. Soc. London, Ser. A., 357, 1761

Pälike, H. 2002, Thesis, University of Cambridge

Pälike, H., \& Shackleton, N. J. 2001, EPSL, 400, 1029

Pälike, H., Laskar, J., \& Shackleton, N. J. 2004, Geological constraints on the chaotic diffusion of the Solar System, Geology, in press

Pijpers, F. P. 1998, MNRAS, 297, L76

Pilgrim, L. 1904, Versuch einer rechnerischen behandlung des eiszeitenproblems. Jahreshefte fur vaterlandische Naturkunde in Wurttemberg, 60
Poincaré, H. 1893, Méthodes nouvelles de la mécanique céleste, (Paris: Gauthier-Villars), Vol. II

Poincaré, H. 1910, Bull. Astron., 27, 321

Pontécoulant, G. de 1834, Théorie Analytique du Système du Monde, t. III, Bachelier, Paris

Quinn, T. R., Tremaine, S., \& Duncan, M. 1991, AJ, 101, 2287

Rochester, M. G. 1976, Geophys. J. R. A. S., 46, 109

Rubincam, D. P. 1990, Science, 248, 720

Rubincam, D. P. 1995, Paleoceanography, 10, 365

Saha, P., \& Tremaine, S. 1994, AJ, 108 (5), 1962

Sharav, S. G., \& Boudnikova, N. A. 1967a, Bull. I.T.A, XI-4(127), 231

Sharav, S. G., \& Boudnikova, N. A. 1967b, Trud. I.T.A, XIV, 48

Standish, E. M. 1998, JPL planetary and Lunar ephemerides, DE405/LE405. JPL IOM 312.F-98-048, Pasadena

Stockwell, J. N. 1873, Memoir on the secular variations of the elements of the eight principal planets. Smith. Contr. Knowledge, Washington, 18

Sussman, G. J., \& Wisdom, J. 1992, Science, 257, 56

Touma, J., \& Wisdom, J. 1994, AJ, 108, 1943

Varadi, F., Bunnegar, B., \& Ghil, M. 2003, ApJ, 592, 620

Vernekar 1972, Long-period global variations of incoming solar radiation, Meteorol. Monogr., 12

Volland, H. 1978, Earth's rotation from eons to days, ed. Brosche and Sündermann (Springer), 62

Williams, D. M., Kasting, J. F., \& Frakes, L. A. 1998, Nature, 396, 453

Williams, J. G., Boggs, D. H., Yoder, C. F., Ratcliff, J. T., \& Dickey, J. O. 2001, J. Geophys. Res., 106, 27933

Wisdom, J., \& Holman, M. 1991, AJ, 102(4), 128

Yoder, C. F. 1995, Astrometric and geodetic properties of Earth and the Solar System, in ed. T. J. Ahrens, Global Earth Physics: A Handbook of Physical Constants, American Geophysical Union, Washington DC, 1

Zachos, J. C., Shackleton, N. J., Revenaugh, J. S., Pälike, H., \& Flower, B. P. 2001, Science, 292, 274

Zachos, J. C., Pagani, M., Sloan, L., Thomas, E., \& Billups, K. 2001, Science, 292, 686 